
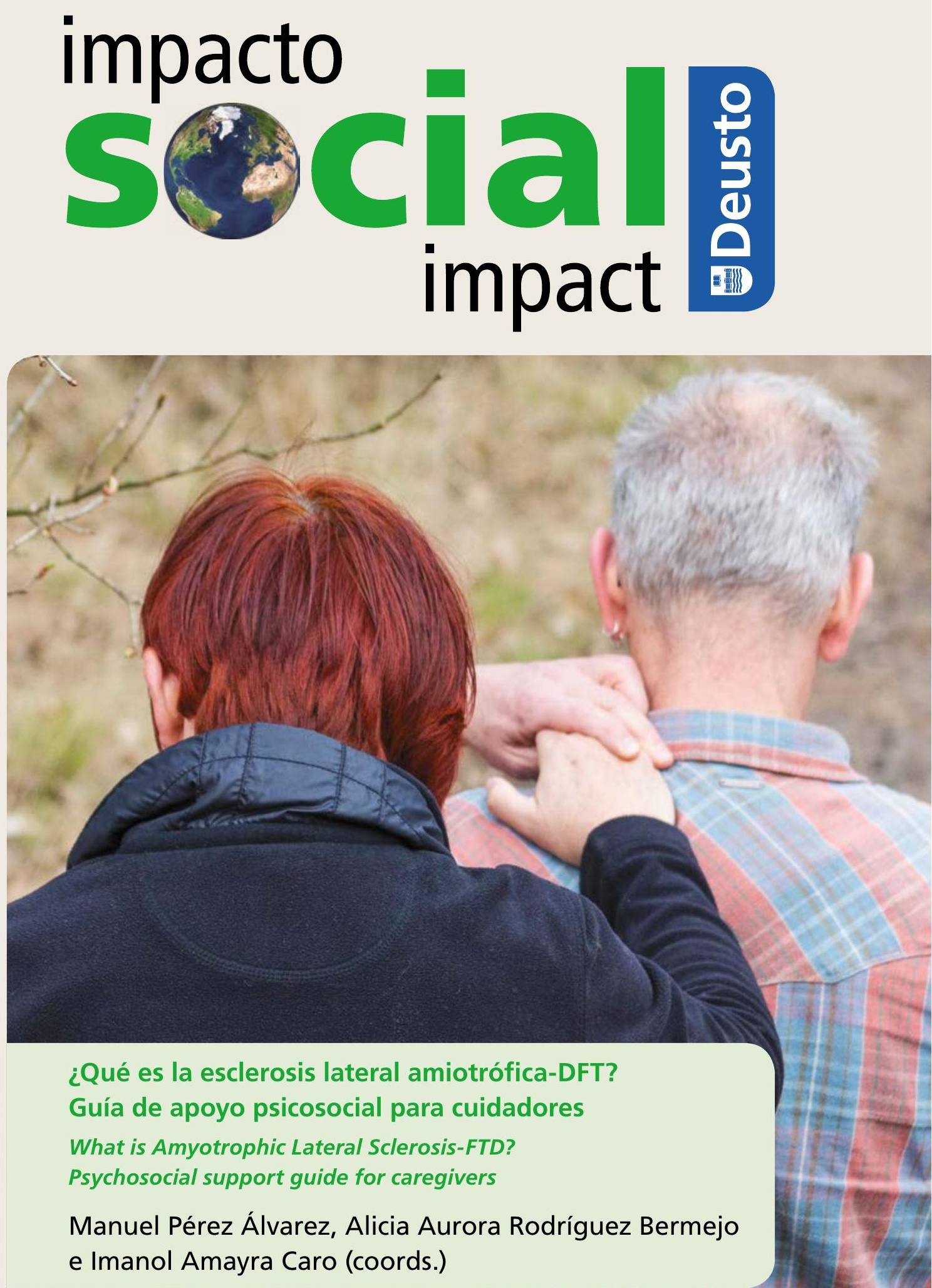



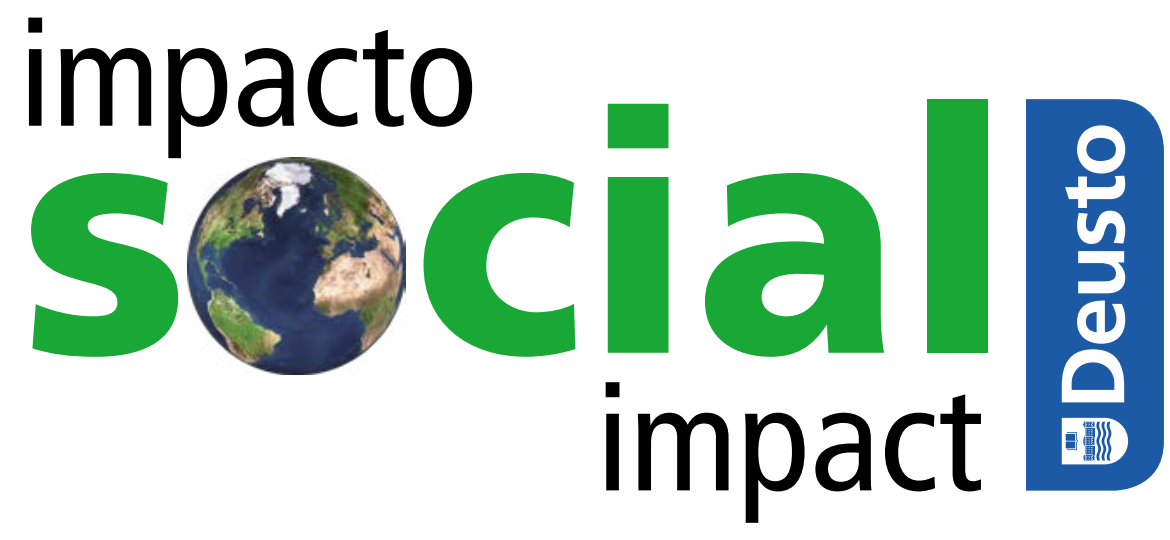


En el marco de la labor llevada a cabo por la Universidad de Deusto (http://www.deusto.es/) en el tema del impacto social de la investigación, anualmente se seleccionan una serie de proyectos de investigación con alto potencial de impacto social, y a partir de ellos, se elaboran y publican los denominados Deusto Social Impact Briefings (DSIB). Son unas monografías breves dirigidas a instituciones sociales, usuarios, policy makers, o empresas que, en lenguaje no académico, responden al objetivo de poner de manifiesto la acción transformadora de la investigación de Deusto, posibilitando que los resultados de la investigación de Deusto sean inteligibles para los agentes sociales y puedan ayudarles a responder a los retos de transformación social a los que se enfrentan, ofreciéndoles buenas prácticas, guías o recomendaciones en la labor que desempeñan.

\section{Frecuencia de publicación y formato}

Deusto Social Impact Briefings se publica electrónicamente y en versión impresa una vez al año. Su primer número se publicó en enero de 2017 como resultado de una convocatoria lanzada a toda la comunidad investigadora en 2016. Este numero corresponde a la convocatoria 2017.

\section{Suscripciones}

Actualmente, no se aplican cargos por la presentación, publicación, acceso en línea y descarga. Pocas copias impresas se ponen a disposición de los colaboradores y socios clave.

\section{Derechos de autor}

Deusto Social Impact Briefings es una publicación de Acceso Abierto de la Universidad de Deusto (España).

Su contenido es gratuito para su acceso total e inmediato, lectura, búsqueda, descarga, distribución y reutilización en cualquier medio o formato sólo para fines no comerciales y en cumplimiento con cualquier legislación de derechos de autor aplicable, sin la previa autorización del editor o el autor; siempre que la obra original sea debidamente citada y cualquier cambio en el original esté claramente indicado. Cualquier otro uso de su contenido en cualquier medio o formato, ahora conocido o desarrollado en el futuro, requiere el permiso previo por escrito del titular de los derechos de autor.
Within the framework of the work carried out by the University of Deusto (http://www.deusto.es/) on the social impact of research, a series of research projects with high potential for social impact are selected annually, and from these, the so-called Deusto Social Impact Briefings (DSIB) are prepared and published as short monographs. They are aimed at social organisations, users, policymakers and businesses. They seek to ensure that research outcomes are intelligible to all these different social actors. They also seek to provide guidelines, best practices and recommendations to support the tasks of meeting the challenges of social transformation that must be faced.

\section{Publication frequency and format}

Deusto Social Impact Briefings is published electronically and in print version once a year. Its first issue appears in January 2017 as result of a call launched in 2016. This issue corresponds to a call launched in 2017.

\section{Subscriptions}

Currently, no charges for submission, publication, online access, and download are applicable. Few print copies are freely made available for key collaborators and partners.

\section{Copyrights}

Deusto Social Impact Briefings is an Open Access publication of the University of Deusto (Spain).

Copyright for this publication is retained by the Publisher. Any part of its content can be reused in any medium or format only for non-commercial purposes and in compliance with any applicable copyright legislation, without prior permission from the Publisher or the author(s). In any case, proper acknowledgement of the original publication source must be made and any changes to the original work must be clearly indicated. Any other use of its content in any medium or format, now known or developed in the future, requires prior written permission of the copyright holder.

(C) Universidad de Deusto

P.O. box 1 - 48080 Bilbao, España

Publicaciones

Tel.: +34-944139162

E-mail: publicaciones@deusto.es

URL: www.deusto-publicaciones.es

ISBN: 978-84-16982-48-6 (version impresa / printed version)

Deposito Legal / Legal Deposit: BI-108-2017

Impreso y encuadernado en España / Printed

and bound in Spain 


\section{Dirección y Coordinación Editorial}

Rosa Maria Santibañez Gruber, Universidad de Deusto, España

Antonia Caro González, Universidad de Deusto, España

\section{Comité Cientifico:}

Javier Arellano Yanguas, Director del Centro de Ética Aplicada e investigador principal del equipo «Ética Aplicada a la Realidad Social» (EARS), Universidad de Deusto.

Antonia Caro González, Directora de la Oficina de Proyectos Internacionales de Investigación y experta en temas europeos, innovación en gestión de la investigación e impacto social, Universidad de Deusto, España.

Laura Teresa Gómez Urquijo, investigadora y Profesora en la Facultad de Derecho e investigadora del equipo Desarrollo Social, Economía e Innovación para las Personas (EDISPE), Universidad de Deusto, España.

Amaia Méndez Zorrilla, Profesora en la Facultad de Ingenieria e investigadora del equipo E-vida, Universidad de Deusto, España.

José Javier Pardo Izal, Profesor del departamento de Teología e investigador en Teología Bíblica y su influencia cultural, Universidad de Deusto, España.

Rosa María Santibañez Gruber, Vicerrectora de Investigación y Transferencia e investigadora principal del equipo Intervención: Calidad de Vida e Inclusión Social, Universidad de Deusto, España.

\section{Comité Asesor Externo:}

Isabel Domínguez Elías, Directora Colegio Esclavas SC Fátima Ikastetxea (Fundación Educativa $A C I$ ), España.

Igotz López Torre, Alcalde de Zamudio, España.

Rafael Mendia Gallardo, Zerbikas Fundazioa, España.

D. José Félix Pérez Tolosa, Presidente de la Asociación de Demencia Frontotemporal, España.

\section{Editors}

Rosa Maria Santibañez Gruber, University of Deusto, Spain

Antonia Caro González, University of Deusto, Spain

\section{Scientific Committee:}

Javier Arellano Yanguas, Head of the Centre for Applied Ethics and Main Researcher in the Ethics Applied to Social Reality research team at the University of Deusto, Spain.

Antonia Caro González, Head of the International Research Project Office and expert in European issues, social impact and innovation in research management at the University of Deusto, Spain.

Laura Teresa Gómez Urquijo, lecturer at the Faculty of Law and researcher in the Economics, Social Development and Innovation for People (EDISPE) team at the University of Deusto, Spain.

Amaia Méndez Zorrilla, lecturer at the Faculty of Engineering and researcher in the E-Life team at the University of Deusto, Spain.

José Javier Pardo Izal, lecturer at the Department of Theology and researcher working on biblical theology and its cultural influence at the University of Deusto, Spain.

Rosa María Santibañez Gruber, Deputy Rector for Research and Knowledge Transfer and Main Researcher in the Intervention: Quality of Life and Social Inclusion team at the University of Deusto, Spain.

\section{External Advisory Board:}

Isabel Domínguez Elías, Director of Esclavas SC - Fátima School (Fundación Educativa ACI), Spain.

Igotz López Torre, Mayor of municipality of Zamudio, Spain.

Rafael Mendia Gallardo, Zerbikas Fundazioa, Spain.

D. José Félix Pérez Tolosa, President of Frontotemporal Dementia Association, Spain.

Oficina Editorial / Editorial Office:

DEUSTO Social Impact Briefings

International Research Projects Office (IRPO)

Universidad de Deusto

Avda. Universidades 24

48007 Bilbao

Tel: +34944139000 (ext 2136)

Email: Ms. Barbara Rossi <barbara.rossi@deusto.es>

Web: http://www.deusto.es/ 



\section{Prólogo}

A través de los 'Deusto Social Impact Briefings' (DSIB) la investigación en Deusto (http:// www.deusto.es/) persigue trasladar el impacto de sus resultados más allá del mundo académico e incidir, de acuerdo con la misión de la Universidad, en la transformación de la sociedad actual. La Universidad entiende como investigación con impacto social aquella que contribuye a procesos de transformación hacia sociedades más justas y diversas, en las que prime el desarrollo y bienestar social inclusivos y realizada en diálogo con agentes sociales, entidades y/o empresas.

Los Deusto Social Impact Briefings (DSIB) son monografias breves que permiten mostrar las capacidades de investigación instaladas en Deusto a través de proyectos desarrollados por equipos de nuestra universidad en las áreas de conocimiento de alta relevancia social. Publicados en papel (tanto en castellano como en inglés) con una periodicidad anual, se pueden encontrar también online en la página web de Deusto Research.

Las publicaciones están dirigidas a entidades sociales, usuarios, policy makers y/o empresas, posibilitando que los resultados de las investigaciones sean inteligibles para estos diferentes agentes sociales y pretenden, mediante la oferta de buenas prácticas, guías o recomendaciones, apoyarles en la labor que desempeñan para responder a los retos de transformación social a los que se enfrentan.

Dentro del proyecto estratégico Deusto 2018 y en el marco del Plan Director de Impacto Social, el presente briefing fue seleccionado por su alto potencial de impacto social, a través de una convocatoria lanzada a toda la comunidad investigadora en 2017. Forma parte de un conjunto de cuatro briefings resultantes de la mencionada convocatoria y que conforman la segunda edición de los DSIB.

El briefing ¿Qué es la esclerosis lateral amiotrófica-DFT? Guía de apoyo psicosocial para cuidadores es una guía de referencia para las familias afectadas, las asociaciones de apoyo, el personal de asistencia, médico y de enfermería involucrados en este proceso de creciente interés científico y social. Este briefing supone un primer acercamiento a la realidad de esta enfermedad y repasa los principales hallazgos científicos al tiempo que proporciona pautas de ayuda psicosocial prácticas enfocándose en el arduo rol del cuidador.

Queremos agradecer el esfuerzo conjunto del personal investigador y de los agentes sociales en la compleja tarea de repensar los resultados de las investigaciones realizadas en un formato más accesible, esperamos que útil, y que cumpla el potencial impacto social que perseguimos mediante su uso y aplicación por profesionales, ciudadanos, agentes públicos y sociales que trabajáis en los temas.

Agradeciendo de antemano el interés por esta iniciativa quedamos a la espera de nuevas propuestas, sugerencias y comentarios que nos ayuden a mejorar de cara a ediciones sucesivas.

Rosa Maria Santibañez Gruber, Dirección DSIB Antonia Caro González, Dirección DSIB

Marzo 2018 


\section{Foreword}

Through the Deusto Social Impact Briefings (DSIB), researchers at the University of Deusto (http://www.deusto.es/) seek to convey the impact of their research results beyond purely academic circles and, in line with the mission of the University, to help transform today's society. For the University, "research with social impact" means research that helps to transform society into a more just, more diverse form where the emphasis is on inclusive development and social welfare through dialogue with social actors, organisations, and/ or businesses.

The Deusto Social Impact Briefings (DSIB) are brief monographic articles that seek to highlight the research capabilities at Deusto through projects conducted by teams from the University in knowledge areas highly relevant to society. They are published annually in hard copy (in Spanish and English), and can also be found online on the Deusto Research website.

They are aimed at social organisations, individual users, policy-makers and/or businesses, and seek to turn research outcomes into useful material for these and other social actors. They are intended to provide support in the form of best practices, guidelines and recommendations to help them tackling the challenges of social transformation.

Under the framework of the Master Plan for Social Impact, part of the Deusto 2018 strategic project, this briefing was selected on the basis of its high potential for social impact, as part of a call launched to the whole research community in 2017. It is one of a set of four briefings submitted in response to that call, which make up this second Issue of the DSIB.

The briefing What is Amyotrophic Lateral Sclerosis-FTD? Psychosocial support guide for caregivers is a reference guide for families of patients, support associations, medical, nursing and other healthcare personnel involved in this process, which is attracting more and more scientific and social interest. This briefing is an initial examination of the situation of this disease. It covers the main scientific findings and gives practical guidelines for psychosocial care, focusing on the tough role played by care-givers.

We would like to thank all the participating researchers and social actors for their joint efforts in tackling the complex task of rethinking their research outcomes with a view to turning them into a more accessible format, which we hope will help to achieve the social impact that we seek, and will be useful tools for specialists, individual citizens, public-sector, and social bodies working in the relevant field.

We would like to thank everyone for their interest in this initiative and we look forward to receiving further proposals, suggestions, and comments that surely will help us to improve subsequent editions.

Rosa Maria Santibañez Gruber, Editor Antonia Caro González, Editor

March 2018 


\section{¿Qué es la esclerosis lateral amiotrófica-DFT? Guía de apoyo psicosocial para cuidadores}

Manuel Pérez Álvarez, Oscar Martínez Gutiérrez, Esther Lázaro Pérez, Juan Francisco López-Paz, Alicia Aurora Rodríguez Bermejo,

Sarah Berrocoso Cascallana, Maitane García Martín, Mohammad Al-Rashaida, Paula Luna Ovalle, Paula Fernández Elorriaga, Amaia Jometón Elorriaga, Imanol Amayra Caro

1. Introducción

2. Justificación y contexto

3. ELA-DFT: elementos principales $\ldots \ldots \ldots \ldots \ldots \ldots \ldots \ldots \ldots$

3.1. Los problemas relacionados con el diagnóstico: primeros síntomas y las dificultades relacionadas ............... 14

3.2. Aspectos legales y económicos .................. 15

3.3. La comunicación con el afectado, con niños y con otras personas................................ 16

3.4. El cuidador: el eje central de la asistencia del afectado ..... 20

3.5. Aceptar la enfermedad: la resiliencia ................ 21

3.6. El respiro del cuidador....................... 24

3.7. Solicitar ayuda a otras personas ................. 27

3.8. La psicoeducación del cuidador .................. 28

3.9. Resultados de estudios con cuidadores de ELA-DFT . . . . . 28

4. Conclusiones........................ 30

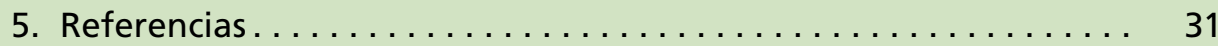

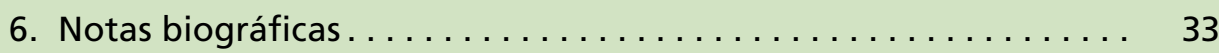





\title{
¿Qué es la esclerosis lateral amiotrófica-DFT? Guía de apoyo psicosocial para cuidadores
}

\author{
Manuel Pérez Álvarez, Oscar Martínez Gutiérrez, Esther Lázaro Pérez, \\ Juan Francisco López-Paz, Alicia Aurora Rodríguez Bermejo, \\ Sarah Berrocoso Cascallana, Maitane García Martín, Mohammad Al-Rashaida, \\ Paula Luna Ovalle, Paula Fernández Elorriaga, Amaia Jometón Elorriaga, \\ Imanol Amayra Caro
}

doi: http://dx.doi.org/10.18543/dsib-2(2017)-pp17-45.pdf

\section{Resumen}

La esclerosis lateral amiotrófica (ELA) es una enfermedad que irrumpe bruscamente en la vida de las personas afectadas y sus familiares. Además de las clásicas alteraciones motoras, pueden comprometerse aspectos clave como la cognición, la personalidad y el comportamiento. El carácter imprevisible de estos síntomas supone un desafío físico y psicológico para la persona cuidadora, así como para el resto del entorno social del afectado. El presente briefing supone un primer acercamiento a esta realidad y repasa los principales hallazgos científicos al tiempo que proporciona pautas de ayuda psicosocial prácticas cuando la ELA se presenta con demencia de tipo frontotemporal (DFT). La DFT es responsable de un gran porcentaje de las alteraciones mentales en la ELA y contribuye decisivamente a la sensación de sobrecarga que experimenta el cuidador. Debido a que el foco de interés se sitúa en el cuidador este material puede ser de utilidad para el personal de asistencia, médico y de enfermería involucrados en este proceso de creciente interés científico y social.

\section{Palabras clave:}

Esclerosis lateral amiotrófica, demencia frontotemporal, cuidadores, estrategias de afrontamiento, proceso de enfermedad.

\section{Abstract}

Amyotrophic Lateral Sclerosis (ALS) is a disease that can suddenly change the lives of patients and their families. It can affect not only motor functions but also such key areas as cognition, personality and behaviour. The unpredictability of its symptoms pose both physical and psychological challenges for carers and for everyone else close to the 
patient- This briefing takes an initial look at this situation and outlines the main scientific findings. It also gives practical guidelines for psychosocial care when ALS is associated with frontotemporal dementia (FTD). FTD is responsible for most of the mental disorders associated with ALS, and is a decisive factor when carers feel overwhelmed. The focus of interest here is on care-givers, and the material presented is intended to be of use to medical, nursing and other healthcare personnel involved in this process, which is attracting more and more scientific and social interest.

\section{Keywords:}

Amyotrophic Lateral Sclerosis, frontotemporal dementia, caregivers, coping strategies, disease process. 


\section{Introducción}

La esclerosis lateral amiotrófica (ELA) es una enfermedad neurodegenerativa caracterizada por debilidad progresiva de miembros superiores e inferiores, de la región bulbar y las funciones respiratorias. La presencia de Demencia Frontotemporal (DFT) en las personas con ELA supone una realidad aún desconocida en muchas ocasiones. La irrupción de esta alteración extramotora supone un desafío para la asistencia y cuidado que proporciona el cuidador: una figura clave en esta enfermedad. Esta guía propone distintas estrategias e información que puede ser de utilidad para las personas involucradas en este proceso.

Basados en trabajos internacionales y en investigaciones realizadas desde la Universidad de Deusto, este briefing recoge la definición, los principales síntomas y los aspectos básicos de la enfermedad, haciendo hincapié sobre:

a. Las particularidades que presenta para el cuidado de estos enfermos.

b. La comunicación que se dará con el paciente, con los posibles menores implicados (p. ej., los hijos del paciente) y con otras personas externas.

c. El rol del cuidador y el manejo del día a día.

\section{Justificación y contexto}

La siguiente guía presenta información acerca del complejo ELA-demencia frontotemporal. En España existen alrededor de 600.000 personas con demencia y cerca de 400.000 con enfermedad de Alzheimer (de Pedro-Cuesta, Virués-Ortega, Vega, Seijo-Martínez, Saz, Rodríguez y Martínez-Martín, 2009). Se estima que en España esta enfermedad afectará a casi 600.000 personas en 2030 y que en el año 2050 llegue al millón de afectados (Sánchez et al., 2006).

Por su parte la ELA es una enfermedad de tipo neuromuscular progresiva que consideraba la alteración motora como la única manifestación de los signos y síntomas asociados. Hoy en día la ELA es entendida como una enfermedad heterogénea entre cuyos componentes

Estas cifras han situado a la demencia como una de las principales prioridades sociales, económicas y de desarrollo del país puede encontrarse frecuentemente el desarrollo de una demencia de tipo Frontotemporal (DFT) (Wilson, Grace, Munoz, He y Strong, 2001).

La DFT se manifiesta por una serie de alteraciones variadas que pueden ir lentamente deteriorando la vida social por la conducta extraña, la pérdida de habilidades sociales y los cambios de la personalidad. Se comienza a sustituir el interés por las cosas debido a la pasividad (p. ej., sus aficiones) y dificultad para seguir una conversación. Complementariamente se 
producen cambios de humor; se enfadan fácilmente, comienzan a aislarse de sus relaciones sociales, prefieren quedarse más en casa por la mayor sensación de control al ser un entorno conocido.

En relación con el cuidador primario, la falta de un apoyo social adecuado es uno de los factores que incrementan la sobrecarga (Prieto, Eimil, López de Silanes, Llanero y Villarejo, 2017). El cuidador de una persona con ELA-DFT se enfrenta a una serie de desafíos que ponen a prueba sus límites emocionales y psicosociales.

El principal objetivo de esta guía es aportar información valiosa acerca de esta enfermedad, dándose a conocer la posible evolución que tendrá el paciente desde las fases tempranas de aparición de los sintomas, durante la enfermedad, hasta el final de la vida.

Las demencias tienen un alto impacto social tanto en la persona afectada como en su entorno. Según datos del Instituto Nacional de Estadística, las tasas de prevalencia para mayores de 65 años se sitúan entre el $5.2 \%$ y el $16.3 \%$, llegando al $22 \%$ en varones mayores de 85 años y al $30 \%$ en mujeres del mismo rango de edad. En el caso de los pacientes de ELA, la demencia se presenta en un $15 \%$ de los enfermos, comúnmente alrededor de los 60 años (Lillo, Savage, Mioshi, Kiernan y Hodges, 2012).

En el caso de los pacientes con ELA se trata de una demencia frontotemporal (DFT) que se caracteriza por cambios de personalidad, irritabilidad, y alteraciones del pensamiento y del lenguaje que han dado lugar a que hoy se considere una variante bien diferenciada de la ELA: la ELA-DFT (Strong, Abrahams, Goldstein, Woolley, Mclaughlin, Snowden y Rosenfeld, 2017). En la actualidad se investiga la proporción de pacientes que tienen esta variación y los factores y genes involucrados. Por el momento, los datos parecen indicar que los pacientes de ELA-DFT son menos compatibles con los tratamientos recomendados y que tienen una supervivencia más corta que los enfermos sin el componente de demencia (Govaarts, Beeldman, Kampelmacher, van Tol, van den Berg, van der Kooi y de Haan, 2016).

Las alteraciones comportamentales más sobresalientes de la ELA-DFT son la apatía, seguida por la desinhibición. En estos casos los pacientes no son conscientes de los cambios que están ocurriendo. También es frecuente que se presenten alteraciones cognitivas Ilamadas disejecutivas; es decir, la incapacidad para organizar los medios y prever las consecuencias de las acciones, así como alteraciones del lenguaje (Murphy, Factor-Litvak, Goetz, Lomen-Hoerth, Nagy, Hupf y Bedlack, 2016; Strong, 2008). 


\section{ELA}

\section{esclerosis lateral amiotrófica}

Una enfermedad degenerativa de tipo neuromuscular

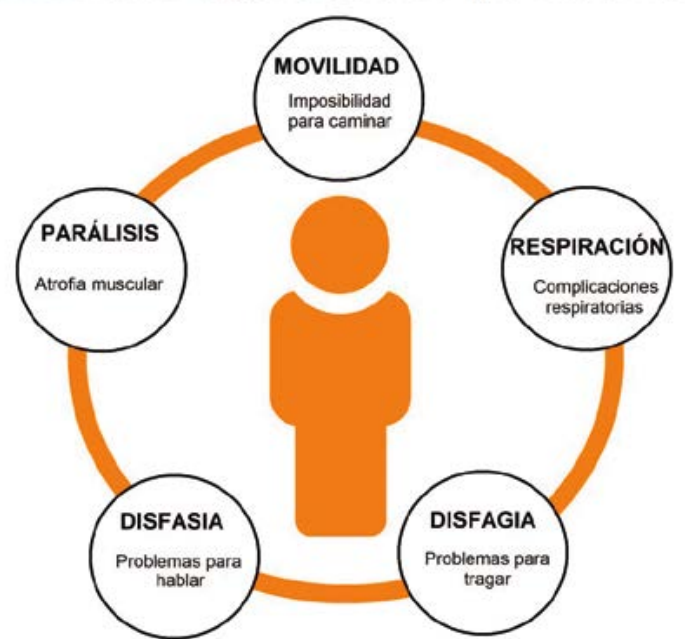

\section{ELA en datos}

- La media de edad en la que se presentan los síntomas es de 60,5 años.

- El 5-10\% de los casos son formas familiares, que se heredan principalmente de manera autosómica dominante.

- Se presenta más frecuentemente en hombres que en mujeres, con una proporción de 1.78/1.

- En España, la prevalencia es de 3,5 casos cada 100000 habitantes. Es decir, existen alrededor de 4000 casos.

La ELA es una enfermedad grave cuyo curso y desarrollo requiere de la presencia de personas cuidadoras que se enfrentan a desafíos vinculados a la asistencia de una amplia gama de actividades diarias provocadas por el deterioro en las funciones motoras.

La exigencia de estos cuidados aumenta a medida que la enfermedad progresa y termina por demandar un cuidado a tiempo completo.

Debido a que la enfermedad puede presentarse antes de los 40 años o después de los 70, la persona que desempeñará el rol del cuidador puede ser aquella con 


\section{El impacto de las alteraciones conductuales en la sobrecarga del cuidador es notable respecto al efecto del deterioro cognitivo}

la que convive, padres, cónyuge, hijos o hermanos. La reducida esperanza de vida de los afectados provoca que la carga emocional que debe soportar el núcleo familiar cercano sea inmensa; a esta carga se le debe sumar la gran dependencia física de los afectados y una nueva variable que entra en escena: las alteraciones comportamentales.

\section{ELA-DFT: elementos principales}

\subsection{Los problemas relacionados con el diagnóstico: primeros síntomas y las dificultades relacionadas}

El escenario más común es el de un familiar que se preocupa porque el paciente con ELA está teniendo cambios del humor, o del lenguaje. Tan pronto como empiecen a manifestarse estos cambios es conveniente notificarlos al médico tratante para poner en marcha contingencias tanto para la adaptación de las actividades de cuidado como para el acondicionamiento de la vivienda para prevenir caídas u otros accidentes domésticos.

La evaluación clínica cursa habitualmente con una evaluación por separado del afectado y del cuidador principal. Esto permite a los miembros de la familia revelar sus preocupaciones honestas acerca de cómo el afectado está funcionando en sus actividades cotidianas y en sus relaciones, y facilita la obtención de un buen historial de la aparición y duración de los problemas. Este proceso también ayuda a determinar si hay diferencias en la opinión entre la persona con sospecha de demencia, su familia, amigos o personas cercanas sobre lo que está pasando, o si hay circunstancias sociales o ambientales que pueden ofrecer una explicación alternativa más satisfactoria. De ese modo, la entrevista privada con el paciente generalmente incluye una causa habitual y tratable de deterioro cognitivo, los cuadros ansioso-depresivos y otras causas, incluyendo el posible uso de ciertos medicamentos, y la co-existencia de otros trastornos psiquiátricos.

Prestar atención a la progresión de los síntomas de un ser querido puede ser un hecho complicado; es natural negar o luchar contra estos cambios. Sin embargo, negar o tratar de corregir comportamientos inapropiadamente puede dañar la relación y el cuidado. Por este motivo, la evaluación objetiva ayudará a las personas y al personal de referencia a obtener pautas de guía en actividades de la vida cotidiana. 


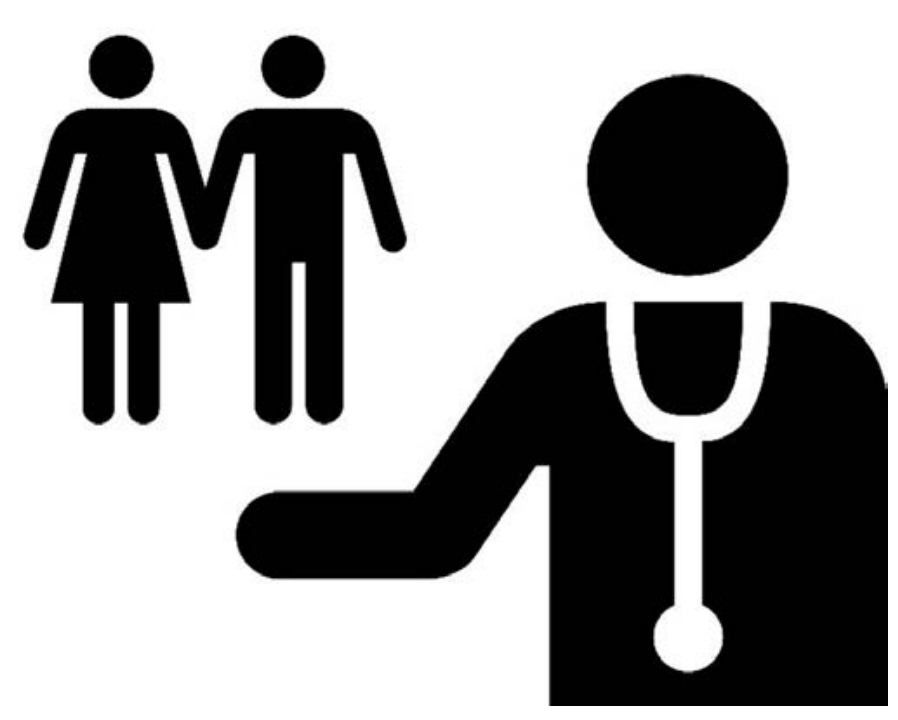

\subsection{Aspectos legales y económicos}

Una vez que se ha detectado la demencia en el paciente con ELA, existen tres posibles acciones que se deben llevar a cabo para preservar al afectado y a su entorno de distintas complicaciones legales que pueden surgir a consecuencia del progresivo deterioro producido por la enfermedad como, por ejemplo, la cuestión del testamento, administración de bienes etc. Es el momento de consultar con un abogado sobre temas de otorgación de poderes, curatela o incapacitación, que se describen brevemente a continuación.

Al otorgar poder, la persona enferma podrá designar a una persona para que le cuide y pueda tener capacidad de decisión acerca de su futuro y el de sus bienes al fallecer. Se trata de una medida que ayuda a prevenir futuros problemas familiares y que debe perseguir el bienestar económico y el mantenimiento del afectado.

La curatela es una de las fórmulas de guarda legal para gestionar el patrimonio del afectado. Este procedimiento solo interviene en los actos que los afectados no puedan realizar por sí solos y en el caso de incapacitados tendrá por objeto la asistencia del curador para aquellos actos que expresamente imponga la sentencia.

En último lugar se sitúa la incapacitación, que es un procedimiento judicial mediante el que se nombra a un tutor supervisado por juez y fiscal para monitorizar actividades económicas y éste es quien tiene obligación de presentar cuentas anuales. 
Hacienda también dispone de distintas ayudas que pueden percibir las personas con un Certificado de Discapacidad igual o superior al 33\%, dependiendo de las Comunidades Autónomas. Los beneficios fiscales pueden incluir alguna o varias de las siguientes prestaciones: a) reducción del IRPF; b) reducción del IVA en la Declaración de la Renta; c) exención de diferentes impuestos de circulación; e) bonificaciones en el impuesto sobre Patrimonio y sobre la Ley de sucesiones y donaciones. Estos beneficios se aplican directamente al afectado, tutores o representantes legales.

\subsection{La comunicación con el afectado, con niños y con otras personas}

La instauración de síntomas comportamentales como la apatía hace que se deban modificar las formas de comunicación con las que nos dirigimos a la persona afectada. La irritabilidad, la agitación o la apatía dificultan de un modo profundo y socavan la comunicación del día a día. Por otro lado, la presencia de menores en el hogar hace necesario poner en marcha distintas estrategias para mejorar las condiciones de las personas implicadas.

\subsubsection{La comunicación con el paciente}

Es importante cuidar la manera en la cual nos dirigimos a la persona enferma. Las palabras, el tono de voz, el lenguaje corporal y el sano uso del humor pueden ayudar en el día a día. Se debe evitar tratar a las personas afectadas como niños que han perdido el sentido de sí mismos. Por este motivo, recordar con respeto y gratitud el cuidado que debemos a estas personas puede suponer la motivación necesaria a la hora de ofrecer la atención y el apoyo necesarios para el día a día. En ocasiones, la afectación del lenguaje expresivo, es decir, la manera de comunicarse del paciente, puede verse afectada por las alteraciones de la vocalización causadas por la disartria, deteriorando aún más la comunicación.

Las alteraciones del comportamiento pueden provocar enfados y discusiones en el entorno. Un primer paso para evitar discutir con el enfermo es el desapego. No nos referimos aquí a una indiferencia emocional o fingir que no ocurre ningún problema; el desapego significa aceptar genuinamente a la otra persona tal como es, no como uno desearía que fuera.

Cuando se asume que un ser querido tiene una enfermedad que está afectando a su capacidad de pensar, de reaccionar, y de entender el mundo, puede empezar a comprender el cambio que está ocurriendo, lo cual permite afrontar la situación de un modo menos personal y responder más efectivamente a los retos que el cuidado de una persona con ELA-DFT demanda. Este desapego solo puede hacerse de manera efectiva cuando se realiza cuidadosamente y comienza por 


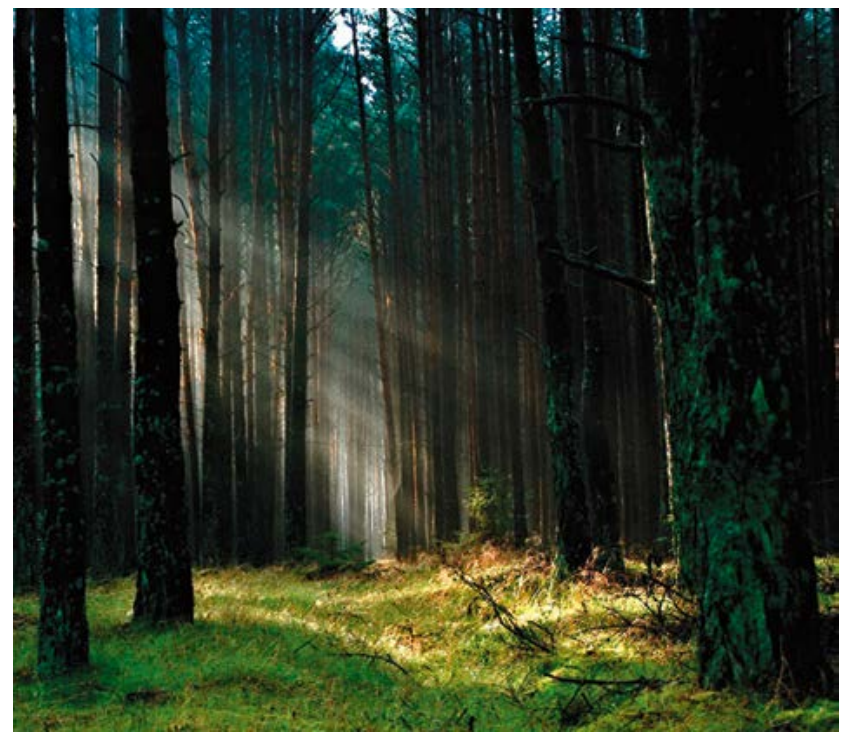

escuchar con respeto al enfermo. Escuchar con respeto implica retener las reacciones emocionales el tiempo suficiente para que la otra persona se sienta escuchada e implica, por otro lado, captar lo que está sucediendo en el interior del paciente. Por ejemplo, si la persona afectada tiene un momento de enfado cuando le mandan tomar los medicamentos puede resultar útil preguntarse: "¿Cómo me estaría sintiendo yo en su situación?" Es posible que la persona enferma esté cansada de que se le diga siempre qué hacer, o que se moleste con el tono de voz o con la forma en la que se le ha preguntado. Tal vez esté irritada porque se le interrumpió durante alguna rutina o porque no entiende realmente para qué son los medicamentos que tiene que tomar.

El desapego en este contexto implica averiguar las emociones que se esconden debajo de las palabras y acciones de la persona enferma y poder responder a esas emociones con comodidad y tranquilidad en lugar de tomar las cosas personalmente.

Cuando la persona está agitada o molesta, re-direccionar la atención de la situación que ha provocado la reacción puede ser una herramienta poderosa. Un primer paso para la distracción consiste en ayudar a hacer algo que sea agradable y placentero (p. ej., poner música, un vídeo que le gusta...) puede hacer que se distraiga y se relaje, generando así distancia con lo que resulta perturbador. También se puede solicitar su ayuda en tareas simples, si la afectación motora lo permite. La distracción también requiere ignorar selectivamente, ya que ayudar a que 
el enfermo se centre en algo agradable implica no prestar atención a lo que está haciendo en ese momento. Esto puede lograrse ignorando sus quejas y luego cambiar el tema a algo más agradable y neutral, como el tiempo que hace afuera, $u$ otras estrategias que pueden funcionar en cada caso.

Al principio, esta herramienta de comunicación puede resultar incómoda de utilizar porque puede parecer infantil o grosero ignorar ciertos comentarios y luego responder con un comentario sin relación; ayudar a que la persona no sienta frustración, no tenga comportamientos agresivos o agitación no es grosero. De hecho, la persona cuidadora está proporcionando una pauta que ayuda al afectado.

Es común que en el transcurso de la enfermedad se produzcan cambios en el entorno familiar y social y puede provocar que algunas personas se distancien. Por distintos motivos, no es raro que las personas con ELA-DFT se opongan fuertemente a recibir ciertos tipos de ayuda de una persona en particular e incluso puede producirse una animadversión hacia el cuidador principal en favor de otras personas del entorno. En tales casos, el camino que ofrece la menor resistencia puede aprovecharse en circunstancias cotidianas como el aseo o la administración de medicamentos. A continuación, se mencionan algunos consejos para la comunicación hacia una persona con demencia:

\begin{tabular}{ll} 
Paciencia & $\begin{array}{l}\text { No tenga prisa. } \\
\text { No pida o pregunte más de una cosa a la vez. } \\
\text { Conceda un instante antes de repetir instrucciones. } \\
\text { Hable lento y claro, no gritando. }\end{array}$ \\
\hline & $\begin{array}{l}\text { Divida las tareas en pequeñas sub-tareas. } \\
\text { Use gestos con la mano para referirse a objetos en particular. } \\
\text { Escriba notas y aclaraciones si son de ayuda. } \\
\text { Tenga en cuenta cómo puede estar sintiéndose la persona ante una situación } \\
\text { de agitación o desgana. }\end{array}$ \\
\hline \multirow{3}{*}{ Empatía } & $\begin{array}{l}\text { Sonría. } \\
\text { Use un tono de voz cálido, amistoso y respetuoso. } \\
\text { El buen ánimo y el sentido del humor pueden ser útiles en momentos difíciles. } \\
\text { Proporcione una felicitación sincera ante algo bien hecho, por sencilla que sea } \\
\text { la tarea. }\end{array}$ \\
\hline Tono & $\begin{array}{l}\text { Evite dar órdenes o actuar de manera autoritaria. } \\
\text { Considere cómo le gustaría ser hablado si fuera usted la persona enferma. }\end{array}$ \\
\hline $\begin{array}{l}\text { Mire directamente a la persona cuando hable o le escuche. } \\
\text { Póngase al mismo nivel cuando se relacionen: si el enfermo está en cama o en } \\
\text { silla de ruedas agáchese hasta hacer un contacto verbal cálido y directo. } \\
\text { Toque delicadamente a la persona para que pueda orientarse hacia usted. }\end{array}$ \\
\hline
\end{tabular}




\subsubsection{La comunicación con los menores}

En ocasiones, la pronta edad en la que se puede presentar la demencia en la ELA puede coincidir con que la persona afectada tenga hijos menores a los que les afecte el proceso y, que representan un colectivo extremadamente vulnerable. En la niñez, cuidar de un padre o madre con ELA-DFT implica una inversión de roles entre padres e hijos. A medida que las capacidades del afectado disminuyen, los menores se pueden encontrar cada vez más en el papel de cuidador. Es importante ser sensible a las necesidades de los hijos durante este tiempo y darles permiso para expresar sus sentimientos.

A medida que la enfermedad progresa y aparecen alteraciones conductuales, ciertos comportamientos pueden provocar vergüenza en los niños y otros sentimientos negativos. En estos casos los menores pueden volcar su enfado y frustración sobre el cuidador principal. Si ese fuera el caso, sería un buen momento para recurrir a los recursos externos que puedan ayudar a lidiar con la gama de emociones que acompañan el tener a un progenitor con ELA-DFT.

Si los menores están en edad escolar conviene notificar al profesorado, la administración y otras personas para que conozcan la situación en el hogar y puedan ser sensibles a la realidad personal del menor. Paralelamente, pueden presentarse problemas en la escuela debido a una serie de razones: los niños que experimentan estas alteraciones en el núcleo familiar y su hogar pueden cuestionar la prioridad y la importancia que los adultos ponen en el rendimiento académico, las tareas escolares y la asistencia escolar. Para evitar esta circunstancia, es importante mantener una vida separada de la realidad de la enfermedad. Disponer de alguien íntimo para la familia puede permitir que los niños tengan la libertad de expresar frustración que no pueden compartir con un familiar cercano que también está afligido.

\subsubsection{Hablar con otras personas acerca de la enfermedad}

Cada situación es diferente y varía con la dinámica familiar, las personalidades, etc. pero, en general, lo mejor es compartir la realidad de la situación con los menores tanto como sea posible. En cualquier caso, el cuidador no debe sentir ninguna presión por comunicar la realidad doméstica ni por acallarla: debe comunicar aquello que desee y reservar cuanta información desee.

A menudo, el proceso de la enfermedad hará que la comunicación con las demás personas se re-ajuste y ponga en perspectiva la voluntad de comunicar con los demás el estado del enfermo y cómo experimenta la enfermedad el cuidador. En ocasiones puede que no se quiera compartir por no recibir reacciones condescendientes, por no querer transmitir sensación de vulnerabilidad, o por discreción. En el proceso de la enfermedad se irán moldeando y adaptando las actitudes hasta 
que las personas cuidadoras puedan hallar la fórmula más adecuadas para sí mismos y para el afectado.

En líneas generales, se asume que cuando la verdad es compartida, la respuesta de los demás es mejor de la que se había anticipado.

\subsection{El cuidador: el eje central de la asistencia del afectado}

¿Cómo relacionarse con una persona físicamente dependiente que no empatiza? Cada cuidado, como cada enfermo, es diferente y puede llegar a ser difícil y estresante cuidar a alguien con ELA-DFT. Supone una serie de desafíos como, por ejemplo:

1. La incertidumbre del avance motor de la enfermedad: desconocer si será un avance rápido o lento.

2. La corta esperanza de vida de las personas con el diagnóstico de ELA.

3. La imprevisibilidad de la conducta del paciente y los problemas que presentan: la falta de inhibición, comportamientos compulsivos y agresividad, o los problemas en el lenguaje.

4. La falta de empatía y emoción del paciente, que a diario menoscaban las fortalezas del cuidador y puede provocar que se sienta desmoralizado, despreciado e irrelevante.

Dada la presencia de estos factores, los cuidadores de pacientes de ELA-DFT están en alto riesgo de depresión, mal humor, insomnio y ansiedad crónica. Además, hay que considerar lo doloroso que es cuidar a alguien cuyo rango emocional puede estar comprometido.

En la mayoría de ocasiones el cuidado diario es realizado por cuidadores informales, principalmente familiares y allegados, responsables de proporcionar cuidados y atender de manera altruista a las personas que presentan algún grado de discapacidad o dependencia.

Dependiendo de los casos, en periodos cortos, los pacientes pasan de una situación de normalidad a una dependencia completa, por lo que necesitan un cuidado específico que la mayoría de las ocasiones es proporcionado por cuidadores del entorno íntimo: madres y padres, cónyuges o hijos. Este cuidado supone un grado elevado de responsabilidad y exigencia, que puede dar lugar a una fuente de estrés y malestar emocional para las personas cuidadoras y los demás miembros de la familia. Esto puede llevar al sufrimiento de consecuencias graves para la salud física y psíquica a raíz de la sobrecarga de cuidado, como depresión o trastornos psicosomáticos. El cuidado es un proceso complejo que mezcla muchas emociones negativas como la culpa, vergüenza, ira, tristeza, confusión e impotencia. Estas emociones se 


\section{Si el cuidador cuenta con}

adecuados recursos externos y

mecanismos de afrontamiento

que faciliten una mejor

adaptación, su rol como

cuidador no tiende a ser

una experiencia frustrante,

pudiendo llegar a ofrecer

aspectos gratificantes presentarán en algún momento y en grado variable. No obstante, los cuidadores que experimentan estos sentimientos no significa que sean malos cuidadores; estas emociones generan estrés y empañan las emociones positivas que pueden despertarse durante el proceso.

Los cuidadores dispuestos a proporcionar el mejor cuidado posible se olvidan con demasiada frecuencia de que también tienen intereses propios y sueños. No es raro que, con la intención de responder a todos los desafíos que se presentan durante la enfermedad, la

persona cuidadora pierda su propia identidad personal y se convierta en una extensión de la persona que cuidan, con el riesgo de enfermar ellas mismas.

Entre los mecanismos que se disponen para afrontar los problemas que intervienen a lo largo del proceso, se encuentran los estilos de afrontamiento: un afrontamiento basado en la emoción está muy relacionado con problemas de salud (ya que modifica o afecta negativamente la evaluación de la situación), mientras que un afrontamiento enfocado hacia el problema se asocia con una reducción del estrés.

Aunque cada proceso es único, algunas reacciones son comunes en el personal cuidador, tales como la impaciencia, irritabilidad, o cansancio. Y se trata en muchas ocasiones de actitudes difíciles de admitir por el propio cuidador o frente a otros e incluso para sí mismo. El problema no es que esas reacciones aparezcan, la dificultad es qué hacer con ellas.

\subsection{Aceptar la enfermedad: la resiliencia}

Las personas que están atrapadas en sus sentimientos son esclavos de las reacciones que tienen. El proceso de ELA-DFT provoca que el afectado ya no sea capaz de ayudarle al cuidador a tomar algunas decisiones y supone una situación dolorosa para todas las personas del entorno: se debe aceptar que una persona que uno conoce íntimamente no es capaz de hacerse cargo de su propia vida. En ocasiones, las personas cuidadoras sienten que están siendo deshonestas o irrespetuosos al censurar lo que dicen a sus seres queridos.

La aceptación de la enfermedad es un aspecto crítico en el cuidador resiliente. 
Ajustar las expectativas de una manera realista puede tomar un tiempo y además se deben reajustar periódicamente debido a la naturaleza progresiva de ELA-DFT. Aceptar las limitaciones es también un proceso de dejar ir; aceptar las propias reacciones internas que surgen frente a la demencia es el ajuste más complejo y difícil de todos. Los cuidadores que abordan su rol con la determinación de manejarlo de una manera idealizada pueden desmoralizarse cada vez que no logran cumplir con sus expectativas personales. En ocasiones extremas puede darse el deseo de "dejar ir" al afectado ante la falta de apoyo y cura para esta enfermedad. Este sentimiento es común y puede aparecer en cualquier momento del proceso. El cuidador debe aceptar este sentimiento y ser consciente del mismo, a pesar de la culpa asociada.

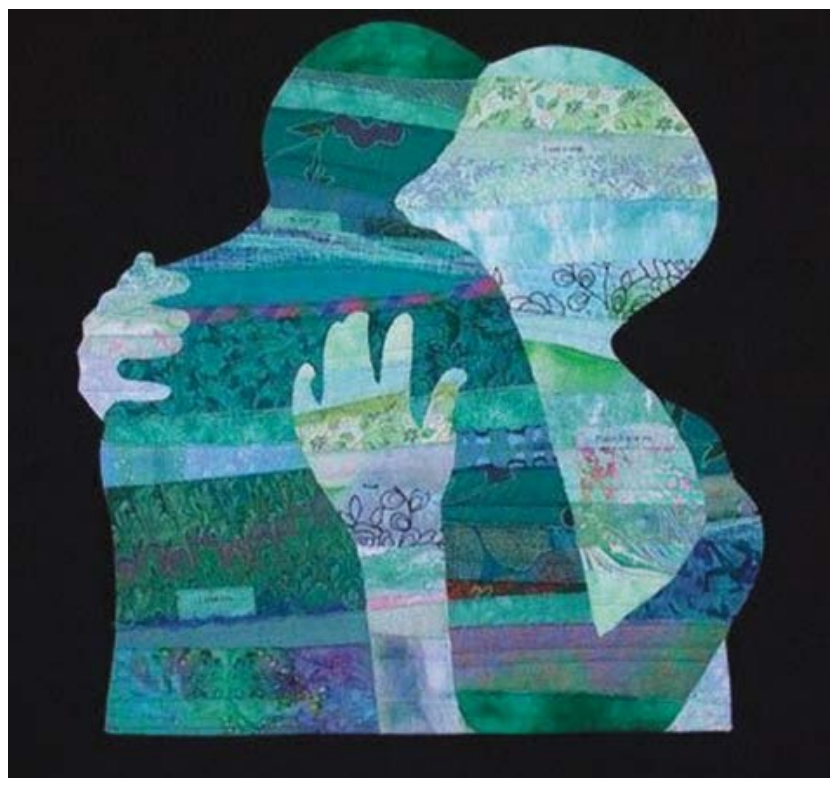

En el ámbito cotidiano, no aceptar la dificultad asociada al cuidado puede retrasar innecesariamente la petición de una ayuda imprescindible. La aceptación implica asumir los altibajos que van a aparecer: no importa la experiencia que posea el cuidador de una persona con ELA-DFT: siempre habrá algo más que aprender. Sin embargo, los cuidadores resilientes aprenden a mantener una visión más saludable del cuidado y confían en que sus valores y principios fundamentales les guiarán, sin importar los retos a los que se enfrentan día a día.

La incapacidad para aceptar la realidad de la ELA-DFT puede socavar los mejores intentos de evitar las discusiones y dificulta la comunicación eficaz. El cuidador que 


\section{La aceptación es un primer paso necesario para poner en marcha los mecanismos de la resiliencia}

utiliza la resiliencia es aquel que entiende profundamente cómo la enfermedad afecta a la persona que está cuidando y que adopta expectativas realistas para su ser querido y para sí mismo. Poner en marcha y dominar estos mecanismos es un proceso de aprendizaje complejo.

Otro aspecto relacionado con la aceptación es asumir las limitaciones que los cuidadores y sus seres queridos pueden experimentar. Cuando las afectaciones motoras no están muy presentes, muchos problemas que experimenta una persona con ELA-DFT pueden ser pasados por alto y en ocasiones es fácil asumir que el afectado todavía es capaz de continuar participando en actividades que realizaba con anterioridad. Una vez que acepte su diagnóstico podrá comprender que algo que está esperando o pidiendo a su ser querido ya no es posible y el cuidador tendrá que empezar a hacer algunas cosas de manera diferente. La aceptación de los nuevos límites también significa tener expectativas realistas para el cuidador y para las personas que pueden ayudar durante el proceso. En ese sentido, es el momento de preguntarse si recurrir a ayuda externa es necesario o razonable; el cuidador debe de mirar alrededor y evaluar de manera realista sus sistemas de apoyo. Probablemente, descubrirá que existen personas en su vida que podrían apoyarlo y que están deseando colaborar en el proceso, aunque otras personas pueden abandonar al afectado y a su familia.

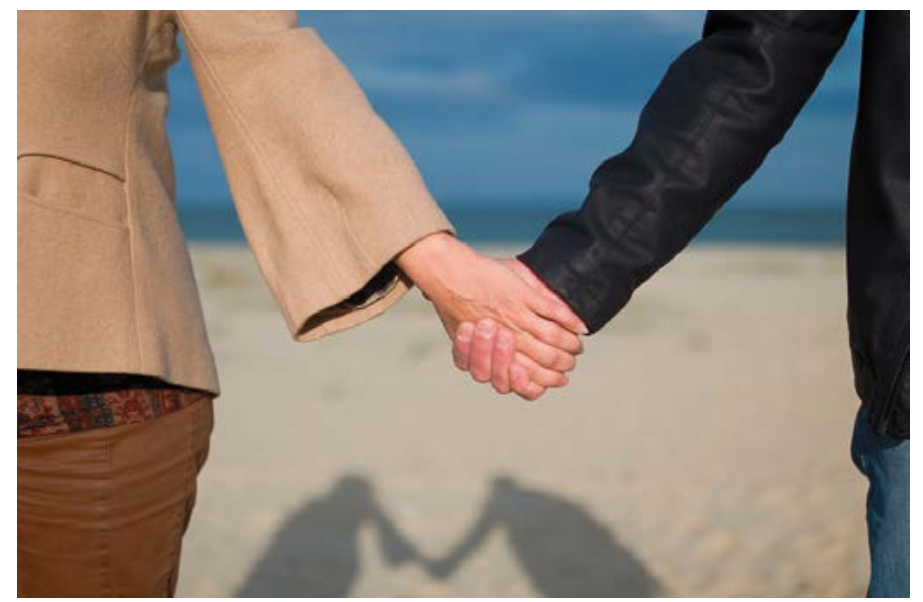

La aceptación es aprender a reconocer los pensamientos, sentimientos, recuerdos y reacciones, es decir, admitir la situación tal como es en realidad. El ver a un 
ser querido incapaz de hacer cosas que hacía fácilmente, es doloroso y difícil de aceptar. De hecho, en muchas ocasiones los cuidadores sienten que aceptar que un ser querido que tiene una enfermedad neurodegenerativa incurable, implica abandonarlo. Quieren que siga luchando y evite o retrase el avance de la enfermedad. Pueden enfadarse cuando eso no sucede y, más tarde, sentirse culpables por enfadarse porque no sucede y luego culpables por enfadarse con alguien que no es responsable de su comportamiento. Es habitual que aparezca un sentimiento de culpa, reflejo de la gran exigencia emocional, y la persona cuidadora puede sentirse avergonzada y frustrada durante el proceso.

Incluso los cuidadores que han aceptado plenamente el hecho de que su ser querido tiene ELA tienen dificultades para aceptar la presencia de severas alteraciones del comportamiento.

Cuando las personas con ELA desarrollan demencia, sus cambios cognitivos y emocionales suelen ocurrir gradualmente, e insisten en que están bien. Una persona con ELA puede tener problemas en el comportamiento que hace semanas no existían. El cuidador tal vez llegue a suponer que estos cambios se producen porque el enfermo está irritado, triste o que está pasando por un mal momento, o incluso que hace ciertas cosas para molestar al cuidador.

Si en el pasado la persona se comportó de manera similar (como en los casos en los que la persona afectada ha experimentado cuadros depresivos). Es importante recordar que, frente a estas experiencias pasadas, los comportamientos actuales no tienen el mismo significado ni las mismas causas. Las personas con demencia son cada vez menos capaces de comprender plenamente las consecuencias de sus actos o no pueden ver cuánta ayuda necesitan de otras personas para realizar algunas tareas. Tienen dificultad para terminar lo que comienzan, para comprender las emociones que provocan en las demás personas o para controlar los cambios rápidos del humor, el ánimo o la agitación que experimentan. Tener esto en consideración no impedirá que el cuidador se enfade o experimente sentimientos de decepción, en la medida en que comprenda que estas actitudes son propias de la enfermedad su manera de afrontarlo será diferente.

\subsection{El respiro del cuidador}

Los resultados de los estudios sobre cuidadores de pacientes ELA-DFT sugieren que cuando estos prestan atención tempranamente a sus necesidades de apoyo social y respiro, mejora su calidad de vida y la del paciente (de Wit, Bakker, van 


\section{¿Qué es el respiro?}

\section{Son intervalos o periodos}

cortos de descanso o alivio
Groenestijn, van den Berg, Schröder, Visser-Meily y Beelen, 2017), por ello todos los cuidadores deben encontrar espacios adecuados para el respiro.

Puesto que cada persona posee diferentes hábitos, gustos e intereses, lo que es respiro para uno puede no serlo para otro. Algunas propuestas para un respiro pueden ser: dar un paseo, visitar amigos, tomar un café, leer el periódico, hacer ejercicio, practicar la meditación, bailar, almorzar o ver películas con amigos.

Es importante, saber qué hacer en los tiempos de respiro y conocer cuándo es necesario. Idealmente, debe ser meditado y puesto en marcha antes de que ocurra la crisis. Al principio, el estrés es leve y a menudo se niegan o ignoran sus mensajes persistentes. Una manera de detectar si el cuidador está padeciendo estrés crónico es prestar atención a señales como los dolores de cabeza y espalda, problemas de estómago y presión arterial alta: la irrupción de estos síntomas puede ser consecuencia de un estrés mal gestionado.

Entre los síntomas depresivos en el cuidador pueden presentarse la incapacidad para dormir, la falta de apetito, la falta de energía y la sensación de "preferir estar en cualquier otro lugar". Entre las manifestaciones clínicas de la ansiedad destacan la incapacidad para concentrarse y crisis de pánico, caracterizados por latidos cardíacos rápidos y respiración agitada. Es habitual que las personas cuidadoras no detecten cuándo se están adentrando en el terreno del agotamiento, aunque el enfermo sí puede notarlo ya que hay una repercu-

Los cuidadores quieren ser

fuertes, y quieren poder

manejar la situación por sí

mismos; pedir ayuda está

reservado sólo para las

circunstancias más desesperadas sión directa sobre la calidad del cuidado: el paciente sabe que el cuidador no está emocionalmente allí. En el asunto del cuidado, como en múltiples aspectos de la vida, la calidad es preferible a la cantidad: el motor de una atención de calidad no puede ser la ira, culpa o resentimiento.

Existen diferentes razones por las que los cuidadores no piden ayuda a pesar de necesitarla. En algunos casos,

puede deberse al deseo de proteger los sentimientos y la dignidad del afectado; no quieren pedir ayuda a familiares $\mathrm{y} / \mathrm{o}$ amigos porque no quieren que sus seres queridos sean vistos padeciendo una enfermedad neurodegenerativa. En otras prefieren que el afectado sea recordado en un mejor momento. Además, los cuidadores no quieren sentir compasión ni el malestar o la vergüenza de terceros que pueden no entender la situación. Por otro lado, los cuidadores también quieren 
proteger al resto de personas que tienen sus propios trabajos, ocio u obligaciones y son reacios a pedir ayuda para no aumentar sus responsabilidades.

Algunas personas anticipan erróneamente que si piden ayuda se les negará o se les dará a regañadientes, lo que de alguna manera parece peor que no pedir ayuda. Por último, cuando el cuidador depende de la bondad de los demás y tiene que solicitar ayuda para realizar tareas que no sean cuidar del afectado, puede justificar prescindir de estas actividades y negarse a realizarlas.

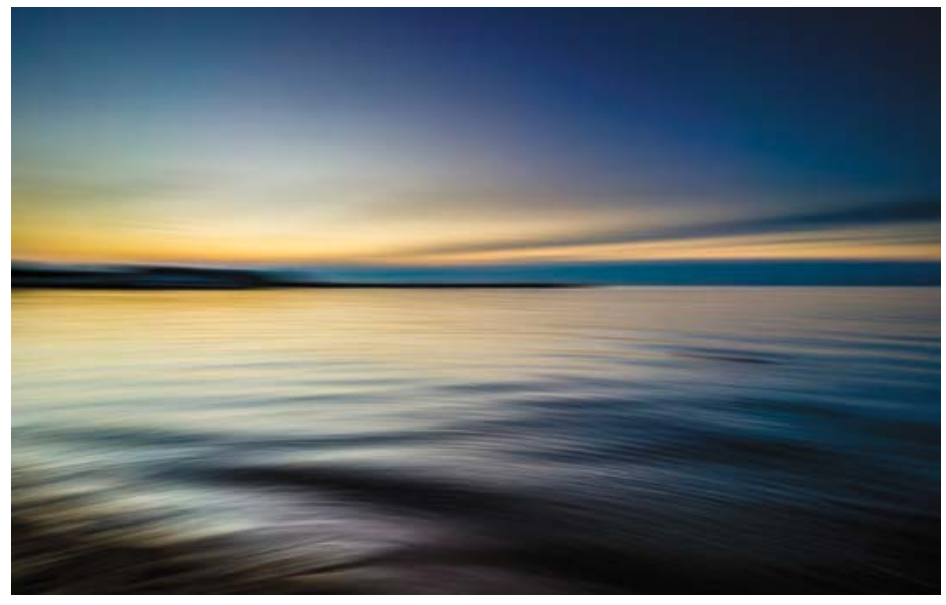

Descuidar la salud física, emocional o espiritual puede perjudicar al cuidador. Al principio, el descuido de la salud no parece marcar una gran diferencia; uno puede sentirse un poco más cansado de lo habitual. Es posible que se descuiden algunas actividades que realizaba con cierta frecuencia como hacer ejercicio, o visitar familiares.

Puede que el cuidador gane o pierda peso, o que su presión arterial se haya incrementado, pero no se siente lo suficientemente mal como para ir al médico. Además, en muchos casos, se suma que los cuidadores también tienen que trabajar fuera de casa y con el tiempo, el estrés prolongado socava la salud física y emocional (Kasuya, Polgar-Bailey, y Robbyn Takeuchi, 2000; Krishnan, York, Backus y Heyn, 2017; Ybema, Kuijer, Hagedoorn y Buunk, 2002).

Pueden aparecer conflictos en el núcleo familiar provocados por la gestión y organización relativas al cuidado del afectado. Estos problemas pueden socavar la moral del cuidador principal de una manera muy acusada por lo que se recomienda la pronta instauración de rutinas de cuidado entre las personas que participen en la tarea, desde la higiene del afectado, hasta los días o momentos de desconexión. 
Una vez que el cuidador toma la decisión consciente de empezar a cuidar de sí mismo, el primer desafío al que se enfrentará es encontrar tiempo para hacerlo: es algo que cada uno tiene que encontrar y no tiene que ser mucho, solamente ser frecuente y regular. El tiempo es un bien precioso para todos y esto es especialmente cierto para los cuidadores de personas con enfermedades neurodegenerativas.

\subsection{Solicitar ayuda a otras personas}

El cuidador tiene que recordar una realidad: cuidar de un ser querido no reemplaza la necesidad de hacer regularmente algo que uno ama, inspira y llena de energía. Hacer algo que uno ama, a diario, ayuda a mantenerse en contacto con la parte creativa, curiosa y que da significado a la vida. Si un cuidador quiere incorporar la facultad de resiliencia debe cultivar estos aspectos.

Los servicios de cuidadores formales, como los programas de salud para adultos y el cuidado en el hogar, han ayudado a muchos cuidadores y sus seres queridos. Sin embargo, no todo el mundo quiere o es capaz de usarlos. Para estos cuidadores, el respiro puede provenir de ayuda de familiares y amigos. De hecho, incluso aquellos cuidadores que usan servicios formales casi siempre necesitan diferentes tipos de asistencia además de los que proporcionan los dispositivos de salud y los cuidadores formales. Los pacientes y los cuidadores tienen personas en sus vidas que los aman y quieren ayudar, pero que simplemente no saben cómo hacerlo.

Por otro lado, es frecuente que no sepan qué ayuda solicitar de los demás. Cuando se solicita ayuda a otra persona nos enfrentamos a la posibilidad de que respondan "no". A ello se le puede sumar que el cuidador se sienta criticado por no ser capaz de sobrellevar la situación por su cuenta. En otros casos, es posible que no sepa qué decir a las personas que no conoce mucho, porque no tiene la confianza necesaria; y a las personas más cercanas puede resultar más complicado de lo que parece solicitar ayuda. La otra gran razón por la que los cuidadores a menudo no piden ayuda es que simplemente no saben qué tipo de ayuda realmente necesitan o a quién deben preguntar.

Un modo para averiguar qué tipo de ayuda se necesita es hacer una lista. Por ejemplo, uno puede preguntarse: ¿Cómo es un día normal para mí? ¿Necesito ayuda para preparar comidas, comer, vestir o duchar al afectado? ¿Necesito realizar un recado y no tengo tiempo para ir a comprar? ¿Qué tareas están pendientes en la casa? Si uno repara en estos puntos descubrirá que algunas cosas que necesitan de la ayuda de otra persona son puntuales, domésticas, o estacionales, como hacer una limpieza general. Otras pueden ser más personales, pero igualmente necesarias, como tener unas pocas horas de respiro cada sábado por la mañana.

Con el tiempo el cuidador puede encontrar que estas situaciones no tienen tanta importancia, o que no compensa solicitar la ayuda por el tiempo que 
requerirá explicar qué necesita y tal vez tenga que compensar esta ayuda en el futuro. Algunos cuidadores no tienen la claridad para reconocer las necesidades que emergen y además puede resultar difícil saber qué ayuda solicitar a qué persona. Una buena manera de averiguar las necesidades es hacer una lista de las personas con las que tiene contacto en su día a día. Una lista típica incluiría a familiares, vecinos, amigos, compañeros de trabajo u otros miembros de las organizaciones de servicios, a pesar de que habrá personas que hayan desaparecido durante el curso de la enfermedad. Ahora, tome la lista de las actividades para las que necesita ayuda y cuál se podría delegar. Compare ambas listas y con seguridad verá que existen personas que se ajustan mejor a algunas tareas, y que otras personas encajan más en otros tipos de ayudas. Algunas veces puede que no haga falta una confianza muy afianzada para solicitar un recado de la farmacia, y otras requerirá de un trato más estrecho para pedir que se hagan cargo del paciente para poder tener una mañana de respiro.

\subsection{La psicoeducación del cuidador}

La psicoeducación es un método para proporcionar información de calidad sobre diversos aspectos de una determinada enfermedad, como los síntomas, el curso, las consecuencias o el pronóstico. También para disipar conocimientos falsos o mitos, enseñar las diversas opciones de tratamiento disponibles y ayudar a identificar las señales que puedan indicar un empeoramiento. La psicoeducación es un proceso en el que se intentan mejorar las habilidades de cuidador mediante el suministro bidireccional de información, no centrándose meramente en la provisión de información, sino también en el acompañamiento.

En ese sentido, las personas que reciben este tipo de intervención adquieren más conocimientos sobre diversos aspectos de la enfermedad y pueden llegar a identificar signos o síntomas propios de la ansiedad y de la depresión que pueden preceder a las crisis. Proporcionar información sobre la enfermedad comprende uno de los pasos de numerosos programas de intervención. En este sentido, es importante que los clínicos y las asociaciones doten al cuidador de información sobre los síntomas que acompañan a la ELA-DFT, así como identificar los cambios que acontecen en su estado físico o mental para que se pueda adelantar a posibles empeoramientos o crisis y que se produzca un menor impacto de la enfermedad en la vida del cuidador.

\subsection{Resultados de estudios con cuidadores de ELA-DFT}

El cuidado de pacientes con un diagnóstico de ELA-DFT puede llegar a suponer una sobrecarga debido a la sintomatología asociada a la enfermedad que sufre el 
paciente y a los problemas asociados. Por ello, se ha investigado cuáles son las intervenciones más adecuadas para reducir la sobrecarga experimentada por los cuidadores, así como conocer cuáles son las características que definen a aquellos cuidadores mejor adaptados.

Una de las terapias que ha resultado ser satisfactoria para mejorar la calidad de vida de los cuidadores informales es la Terapia Cognitivo-Conductual. Esta terapia tiene como base explicativa que los síntomas y las conductas disfuncionales están mediadas por los pensamientos. De esta forma, la reducción de síntomas psicológicos se puede dar a través de la modificación de creencias y pensamientos disfuncionales. El estudio de Van Froenestikn et al. (2015) demostró que la calidad de vida aumentaba en los cuidadores de pacientes con ELA tras la intervención, y que tenía un efecto moderado sobre la sensación de sobrecarga.

La Terapia de la dignidad se desarrolló para reducir el estrés psicosocial y existencial derivado de la pérdida de un ser querido. Esta terapia requiere que el cuidador recuerde aspectos de su vida que quiere conservar, se le ayuda a encontrar propósitos u objetivos en su vida, y a expresar palabras en el contexto de una enfermedad terminal. Estudios como el de Bentley, Aoun, O'Connor, Breen, y Chochinov (2012) resaltan la importancia que tiene llevar a cabo este tipo de terapias en familiares y cuidadores de ELA-DFT, ya que parece tener un impacto positivo sobre la calidad de vida.

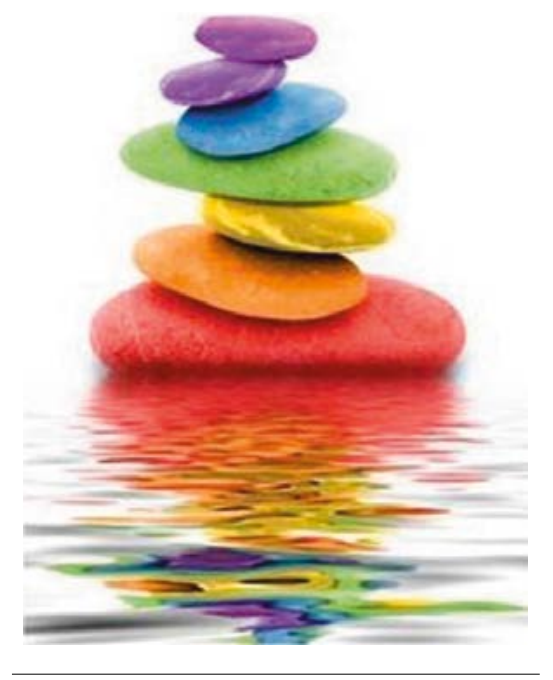

La meditación basada en mindfulness ha sido probada en muchas enfermedades crónicas, obteniendo como resultado una reducción del estrés en la mayoría de los casos. Esta técnica se basa en la meditación proveniente de la filosofía budista y su enfoque se basa en la atención plena. La atención plena consiste en prestar atención al momento presente, de forma consciente, y sin juzgar lo que esté ocurriendo. Esta práctica va a permitir a la persona una mayor consciencia de qué es lo que está ocurriendo en su cuerpo y, consecuentemente, se va a conseguir un mejor balance de las emociones y una mejor estabilidad emocional, que va a ser una buena base para reducir el estrés y lograr una mayor resiliencia. El grupo de Pagnini, Di Credico, Gatto, Fabiani, Rossi, Lunetta y Banfi (2014) llevó a cabo esta intervención en pacientes que sufrían ELA y supone una buena herramienta, especialmente durante las primeras etapas de la enfermedad.

En cuanto a las intervenciones psicológicas efectivas para cuidadores de pacientes con un diagnóstico de DFT, se ha visto que los grupos compuestos por 
cuidadores, los llamados grupos de apoyo pueden llegar a ser muy beneficiosos. Estos suelen estar compuestos por contenidos educacionales, en los que se les instruye sobre asuntos médicos, legales, financieros y de seguros, e información sobre recursos y servicios. Por otro lado, y una de las cosas más importantes de este tipo de grupos, son los elementos terapéuticos, que consisten en que los cuidadores compartan sus experiencias. Se fomenta el apoyo mutuo, se facilita la expresión de emociones y se desarrollan estrategias de afrontamiento con respecto al manejo de los síntomas conductuales inherentes a la enfermedad. Este tipo de grupos de apoyo han sido vistos por los cuidadores como una ayuda necesaria y sirve para reducir la sobrecarga derivada de los cuidados (Nunnemann, Kurz, Leucht y Diehl-Schmid, 2012).

\section{Conclusiones}

El papel de las personas cuidadoras es integral ya que su labor debe ayudar a mejorar las circunstancias de salud, emocionales y sociales del afectado. Esta tarea es especialmente importante en la ELA debido a la dependencia completa que experimentarán antes o después las personas con este diagnóstico.

Las alteraciones comportamentales, del juicio y del pensamiento no son infrecuentes y suponen un duro reto al que se tienen que enfrentar el entorno directo (cónyuges, hijos/as, padres y madres, amistades) de las personas afectadas. La gran carga y presión, tanto interna como externa, que soportan estas personas hace que habitualmente no reparen en enfoques distorsionados sobre el cuidado que pueden repercutir en la relación cuidador-enfermo, en la labor del cuidado, en su salud y en el entorno.

En muchas ocasiones, el gran abanico de emociones que se presentan durante el proceso hará que la persona cuidadora despliegue igualmente medidas para manejar los sentimientos. Es totalmente comprensible y esperado que el cuidador no siempre gestione bien estas emociones debido a las situaciones demandantes que tiene la ELA-DFT. No reconocer los signos de estar sufriendo una sobrecarga y un proceso de desgaste emocional puede revertirse en un cuidado de menos calidad y en una inferior calidad de vida tanto para la persona enferma como para la cuidadora.

En esta guía se han propuesto algunas estrategias para reconocer estos signos y pautas de apoyo. La realidad clínica y social de la demencia en la ELA comienza a imponerse en el proceso asistencial. Este briefing supone un primer punto de apoyo para las personas cuidadoras de ELA-DFT, así como un material de referencia básico desde los entornos médicos y asociativos. 


\section{Referencias}

Bentley, Brenda, Aoun, S. M., O'Connor, M., Breen, L. J. y Chochinov, H. M “Is dignity therapy feasible to enhance the end of life experience for people with motor neurone disease and their family carers?" BMC Palliative Care 11.1 (2012): 18.

de Pedro-Cuesta, Jesús, Virués-Ortega, J., Vega, S., Seijo-Martínez, M., Saz, P., Rodríguez, F., \&Martínez-Martín, P. "Prevalence of dementia and major dementia subtypes in Spanish populations: a reanalysis of dementia prevalence surveys, 1990-2008." BMC neurology 9.1 (2009): 55.

de Wit, Jessica, Bakker, L. A., van Groenestijn, A. C., van den Berg, L. H., Schröder, C. D., Visser-Meily, J. M. y Beelen, A. "Caregiver burden in amyotrophic lateral sclerosis: A systematic review." Palliative Medicine (2017): 0269216317709965.

Govaarts, Rosanne, Beeldman, E., Kampelmacher, M. J., van Tol, M. J., van den Berg, L. H., van der Kooi, A. J. y de Haan, R. J. "The frontotemporal syndrome of ALS is associated with poor survival." Journal of neurology 263.12 (2016): 2476-2483.

Kasuya, Richard T., Polgar-Bailey, M. P. y MPH Robbyn Takeuchi, M.. "Caregiver burden and burnout a guide for primary care physicians." Postgraduate Medicine 108.7 (2000): 119.

Krishnan, Shilpa, York, M. K., Backus, D. y Heyn, P. C. "Coping With Caregiver Burnout When Caring for a Person With Neurodegenerative Disease: A Guide for Caregivers." Archives of physical medicine and rehabilitation98.4 (2017): 805-807.

Lillo, Patricia, Savage, S., Mioshi, E., Kiernan, M. C. y Hodges, J. R "Amyotrophic lateral sclerosis and frontotemporal dementia: a behavioural and cognitive continuum." Amyotrophic Lateral Sclerosis 13.1 (2012): 102-109.

Murphy, Jennifer, Factor-Litvak, P., Goetz, R., Lomen-Hoerth, C., Nagy, P. L., Hupf, J. y Bedlack, R. S "Cognitive-behavioral screening reveals prevalent impairment in a large multicenter ALS cohort." Neurology 86.9 (2016): 813-820.

Nunnemann, Sabine, Kurz, A., Leucht, S. y Diehl-Schmid, J. "Caregivers of patients with frontotemporal lobar degeneration: a review of burden, problems, needs, and interventions." International Psychogeriatrics 24.9 (2012): 1368-1386.

Pagnini, Francesco, Di Credico, C., Gatto, R., Fabiani, V., Rossi, G., Lunetta, C. y Banfi, P "Meditation training for people with amyotrophic lateral sclerosis and their caregivers." The Journal of Alternative and Complementary Medicine 20.4 (2014): 272-275.

Prieto, C., Eimil, M., López de Silanes, C., Llanero, M. y Villarejo, A. (2017). Ver Informe de la Fundación del Cerebro. Impacto Social de la Enfermedad de Alzheimer y otras Demencias, Actualización. http://www.fundaciondelcerebro.es/docs/IS_EA_y_otras_ demencias.pdf (consultada el 12 de noviembre de 2017).

Strong, Michael J. "The syndromes of frontotemporal dysfunction in amyotrophic lateral sclerosis." Amyotrophic Lateral Sclerosis9.6 (2008): 323-338.

Strong, Michael J., Abrahams, S., Goldstein, L. H., Woolley, S., Mclaughlin, P., Snowden, J. y Rosenfeld, J. "Amyotrophic lateral sclerosis-frontotemporal spectrum disorder (ALSFTSD): Revised diagnostic criteria." Amyotrophic Lateral Sclerosis and Frontotemporal Degeneration 18.3-4 (2017): 153-174. 
Van Groenestijn, A. C., Schröder, C. D., Visser-Meily, J. M., Reenen, E. T. K. V., Veldink, J. H., \& Van Den Berg, L. H. (2015). Cognitive behavioural therapy and quality of life in psychologically distressed patients with amyotrophic lateral sclerosis and their caregivers: Results of a prematurely stopped randomized controlled trial. Amyotrophic Lateral Sclerosis and Frontotemporal Degeneration, 16(5-6), 309-315.

Wilson, C. M., Grace, G. M., Munoz, D. G., He, B. P. y Strong, M. J. "Cognitive impairment in sporadic ALS A pathologic continuum underlying a multisystem disorder." Neurology 57.4 (2001): 651-657.

Ybema, Jan F., Kuijer, R. G., Hagedoorn, M. y Buunk, B. P. "Caregiver burnout among intimate partners of patients with a severe illness: An equity perspective." Personal Relationships 9.1 (2002): 73-88. 


\section{Notas biográficas}

El equipo Neuro-e-Motion, formado por Imanol Amayra (Director), Juan Francisco López Paz, Esther Lázaro, Oscar Martínez, Sarah Berrocoso, Maitane García, Mohammad Al-Rashaida y Manuel Pérez, ha obtenido el 14 de febrero 2018 el primer premio de la XIII edición del Premio UD- Santander de Investigación con el trabajo de investigación "Evaluación e intervención psicosocial en enfermedades raras". El proyecto ganador, liderado por el equipo Neuro-e-Motion, tiene como objetivo principal el desarrollo de estrategias de evaluación e intervención para la mejora de la calidad de vida de personas con enfermedades poco frecuentes y sus familiares, principalmente a través de las tecnologías.

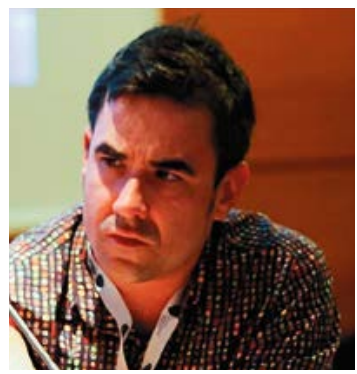

\section{Manuel Pérez Álvarez}

Neuropsicólogo e investigador sobre alteraciones cognitivas en la esclerosis lateral amiotrófica. Máster en Salud Mental y Terapias Psicológicas (Universidad de Deusto).

\section{Oscar Martínez Gutiérrez}

Doctor en Psicología y profesor de la Facultad de Psicología de la Universidad de Deusto. Responsable del programa de aplicación de las TICs en investigación en enfermedades neuromusculares.
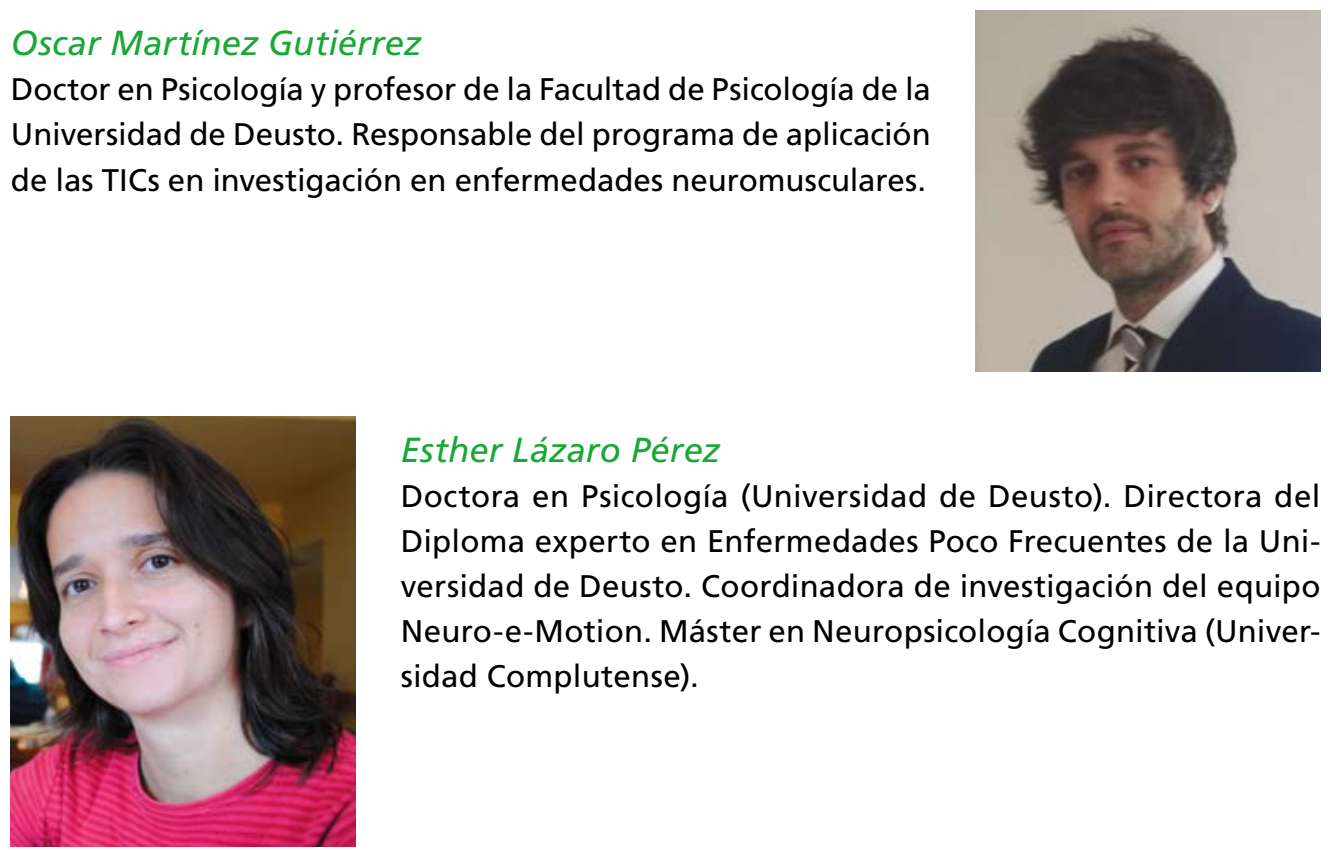

\section{Esther Lázaro Pérez}

Doctora en Psicología (Universidad de Deusto). Directora del Diploma experto en Enfermedades Poco Frecuentes de la Universidad de Deusto. Coordinadora de investigación del equipo Neuro-e-Motion. Máster en Neuropsicología Cognitiva (Universidad Complutense). 


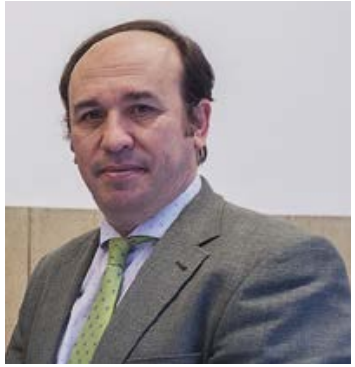

\section{Juan Francisco López-Paz}

Doctor en Psicología y profesor titular de la Facultad de Psicología de la Universidad de Deusto. Profesor en la asignatura “Evaluación e Intervención Clínica en Enfermedades Raras o Poco Frecuentes" (Universidad de Deusto).

\section{Alicia Aurora Rodríguez Bermejo}

Investigadora sobre cuidadores de pacientes con esclerosis lateral amiotrófica. Máster en Neuropsicología Clínica y Psicología General Sanitaria (Universidad de Deusto).
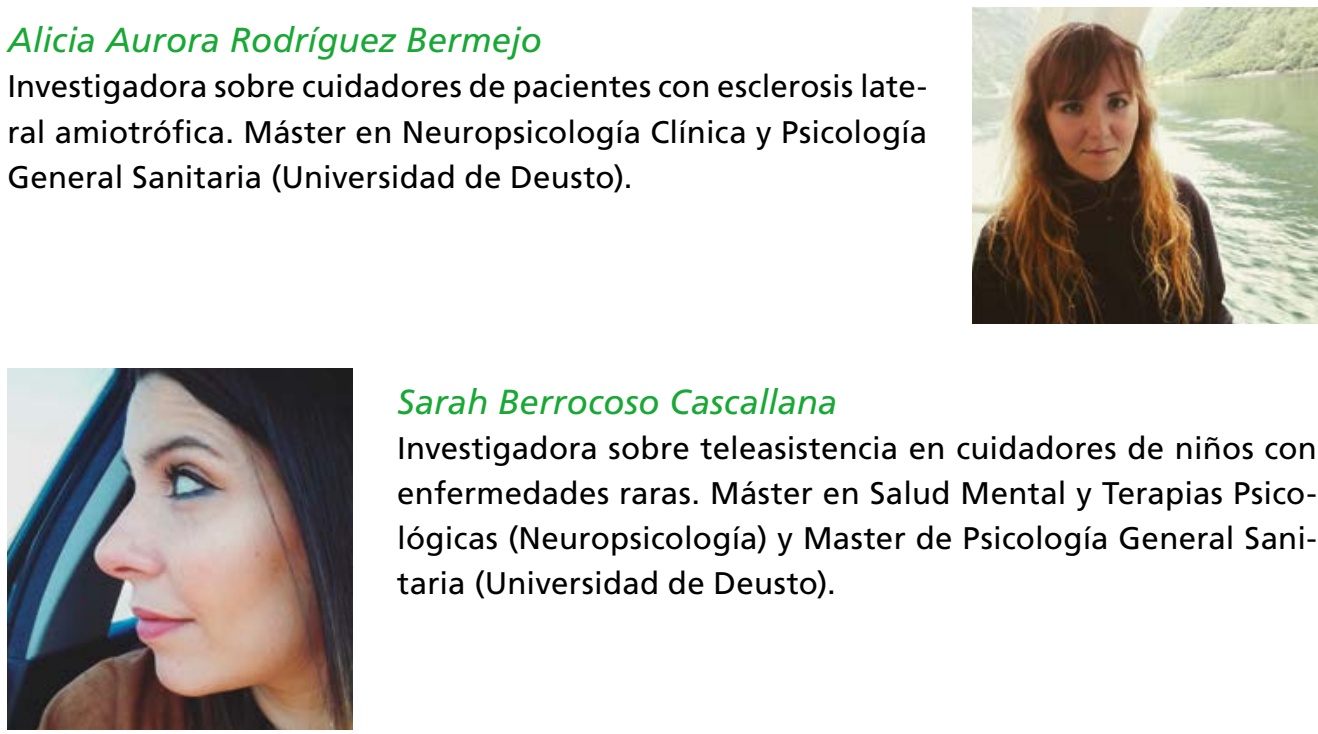

\section{Sarah Berrocoso Cascallana}

Investigadora sobre teleasistencia en cuidadores de niños con enfermedades raras. Máster en Salud Mental y Terapias Psicológicas (Neuropsicología) y Master de Psicología General Sanitaria (Universidad de Deusto).

\section{Maitane García Martín}

Investigadora sobre malformación de Arnold-Chiari. Máster Neuropsicología Clínica y Máster de Psicología General Sanitaria (Universidad de Deusto).
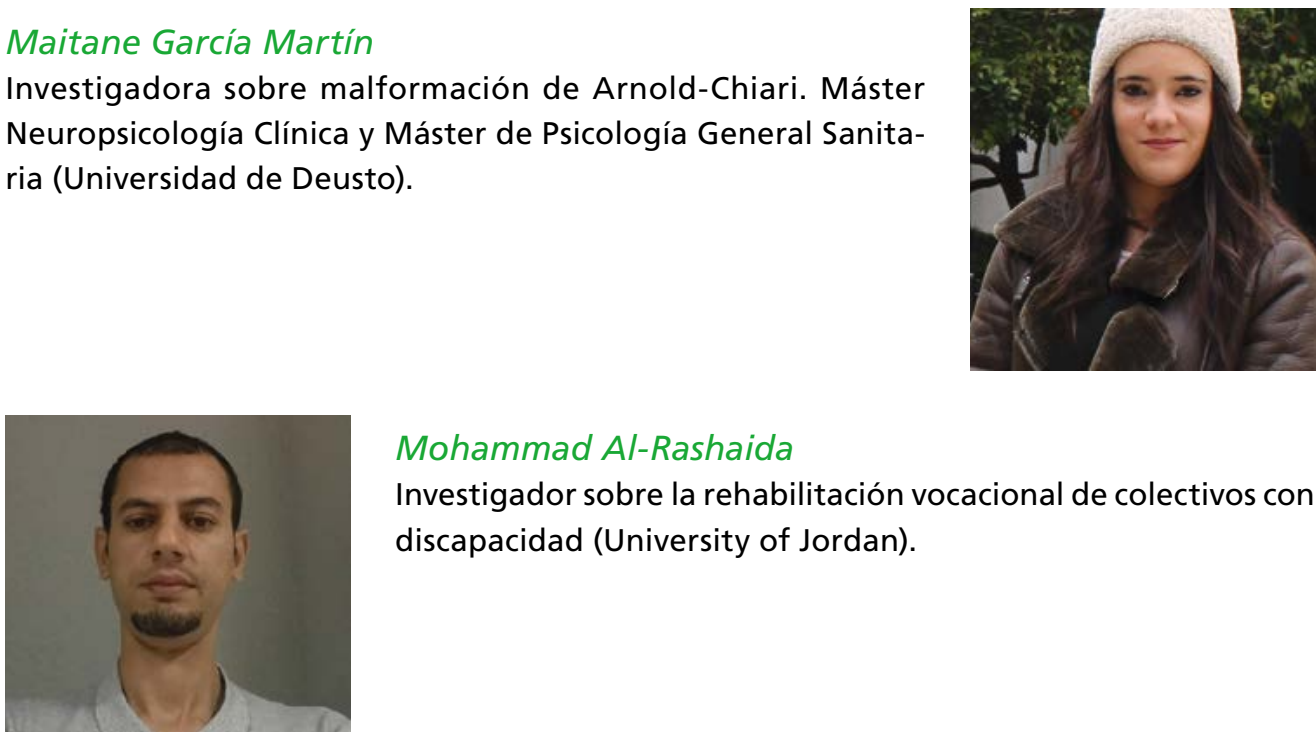

\section{Mohammad Al-Rashaida}

Investigador sobre la rehabilitación vocacional de colectivos con discapacidad (University of Jordan). 


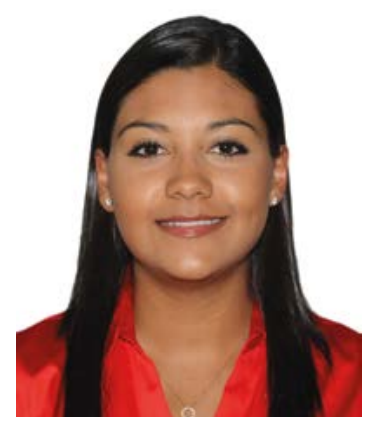

\section{Paula Luna Ovalle}

Psicóloga Clínica (Universidad Francisco Marroquín). Máster en Neuropsicología Clínica (Universidad de Deusto).

\section{Paula Fernández Elorriaga}

Investigadora sobre nuevas tecnologías de la comunicación para personas con demencia. Master en Neuropsicología Clínica y Master en Psicología General Sanitaria (Universidad de Deusto).

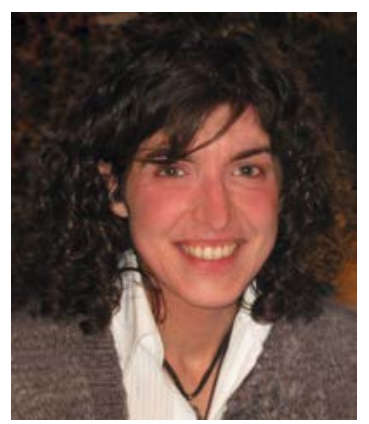

\section{Amaia Jometón Elorriaga}

Psicóloga General Sanitaria especialista en enfermedades raras. Psicoterapeuta especializada en personas con enfermedad neuromuscular.

\section{Imanol Amayra Caro}

Catedrático de Psicología de la Universidad de Deusto. Investigador Principal del equipo Neuro-e-Motion. Jefe del Departamento de Evaluación, Personalidad y Tratamientos Psicológicos de la Universidad de Deusto.

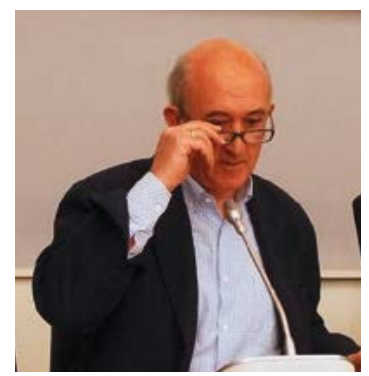





\section{What is Amyotrophic Lateral Sclerosis-FTD? Psychosocial support guide for caregivers}

Manuel Pérez Álvarez, Oscar Martínez Gutiérrez, Esther Lázaro Pérez, Juan Francisco López-Paz, Alicia Aurora Rodríguez Bermejo, Sarah Berrocoso Cascallana, Maitane García Martín, Mohammad Al-Rashaida, Paula Luna Ovalle, Paula Fernández Elorriaga, Amaia Jometón Elorriaga, Imanol Amayra Caro

1. Introduction $\ldots \ldots \ldots \ldots \ldots \ldots \ldots \ldots \ldots \ldots \ldots \ldots \ldots \ldots \ldots \ldots \ldots$

2. Underlying reasons and context.

3. ALS/FTD: Main characteristics . . . . . . . . . . . . . . .

3.1. Diagnosis-related problems: early symptoms and associated difficulties........................... 44

3.2. Legal and financial issues. . . . . . . . . . . . . . . . . . . 45

3.3. Communication with sufferers, with children and with others 46

3.4. The caregiver: the hub of the care system of the patient. . . . 49

3.5. Accepting the disease: resilience. . . . . . . . . . . . 51

3.6. Respite care for caregivers . . . . . . . . . . . . . . 54

3.7. Asking others for help...................... 56

3.8. Psychoeducation of caregivers . . . . . . . . . . . . . 57

3.9. Results of studies involving ALSIFTD caregivers . . . . . . . 57

4. Conclusions. ........................... 59

5. References ......................... 59

6. Biographical notes........................ 61 



\title{
What is Amyotrophic Lateral Sclerosis-FTD? Psychosocial support guide for caregivers
}

\author{
Manuel Pérez Álvarez, Oscar Martínez Gutiérrez, Esther Lázaro Pérez, \\ Juan Francisco López-Paz, Alicia Aurora Rodríguez Bermejo, \\ Sarah Berrocoso Cascallana, Maitane García Martín, Mohammad Al-Rashaida, \\ Paula Luna Ovalle, Paula Fernández Elorriaga, Amaia Jometón Elorriaga, \\ Imanol Amayra Caro
}

doi: http://dx.doi.org/10.18543/dsib-2(2017)-pp47-73.pdf

\begin{abstract}
Amyotrophic Lateral Sclerosis (ALS) is a disease that can suddenly change the lives of patients and their families. It can affect not only motor functions but also such key areas as cognition, personality and behaviour. The unpredictability of its symptoms pose both physical and psychological challenges for carers and for everyone else close to the patientThis briefing takes an initial look at this situation and outlines the main scientific findings. It also gives practical guidelines for psychosocial care when ALS is associated with frontotemporal dementia (FTD). FTD is responsible for most of the mental disorders associated with ALS, and is a decisive factor when carers feel overwhelmed. The focus of interest here is on care-givers, and the material presented is intended to be of use to medical, nursing and other healthcare personnel involved in this process, which is attracting more and more scientific and social interest.
\end{abstract}

\section{Keywords:}

Amyotrophic Lateral Sclerosis, frontotemporal dementia, caregivers, coping strategies, disease process.

\section{Resumen}

La esclerosis lateral amiotrófica (ELA) es una enfermedad que irrumpe bruscamente en la vida de las personas afectadas y sus familiares. Además de las clásicas alteraciones motoras, pueden comprometerse aspectos clave como la cognición, la personalidad y el comportamiento. El carácter imprevisible de estos síntomas supone un desafío físico y psicológico para la persona cuidadora, así como para el resto del entorno social del afectado. El presente briefing supone un primer acercamiento a esta realidad y repasa los 
principales hallazgos científicos al tiempo que proporciona pautas de ayuda psicosocial prácticas cuando la ELA se presenta con demencia de tipo frontotemporal (DFT). La DFT es responsable de un gran porcentaje de las alteraciones mentales en la ELA y contribuye decisivamente a la sensación de sobrecarga que experimenta el cuidador. Debido a que el foco de interés se sitúa en el cuidador este material puede ser de utilidad para el personal de asistencia, médico y de enfermería involucrados en este proceso de creciente interés científico y social.

\section{Palabras clave:}

Esclerosis lateral amiotrófica, demencia frontotemporal, cuidadores, estrategias de afrontamiento, proceso de enfermedad. 


\section{Introduction}

Amyotrophic Lateral Sclerosis (ALS) is a neurodegenerative disease characterised by a gradual loss of strength in the upper and lower limbs, in the bulbar region and in respiratory functions. The presence of frontotemporal dementia (FTD) in ALS patients is a largely unknown area on many occasions. The presence of this extra motor disorder is a challenge to caregivers, who are key figures in dealing with this disease. This guide suggests a number of strategies and sources of information that may be useful to people involved in this process.

On the basis of international studies and research at the University of Deusto, this briefing seeks to define this disease and list its main symptoms and basic aspects, emphasising:

a. particular issues arising from the care of sufferers;

b. communication with patients, with any minors involved (e.g. the patient's children) and other outside persons; and

c. the role of caregivers and how they handle day-to-day events.

\section{Underlying reasons and context}

The guide below provides information on the ALS/FTD complex. In Spain there are around 600,000 people with dementia and almost 400,000 with Alzheimer's Disease (de Pedro-Cuesta, Virués-Ortega, Vega, Seijo-Martínez, Saz, Rodríguez \& Martínez-Martín, 2009). That figure is expected to reach almost 600,000 by 2030 and one million by 2050 (Sánchez et al., 2006).

ALS is a progressive neuromuscular disease whose only signs and symptoms were previously thought to be motor function disturbances. Nowadays it is seen as a heterogeneous disease whose manifestations can frequently include the onset of some kind of frontotemporal dementia (FTD) (Wilson, Grace, Munoz, He \& Strong, 2001).

These figures make dementia one of the top social, economic and development-related priorities for the country
FTD displays different disturbances that can gradually lead social life to deteriorate as a result of erratic behaviour on the part of the patient, resulting in a loss of social skills and changes in personality. Patients begin to lose interest in many things, show a passive attitude to things that they were previously enthusiastic about (e.g. hobbies) and find it hard to follow a conversation. At the same time there are mood changes; patients are quick to anger, begin to cut themselves off from social relationships and prefer to stay at home, where they feel more in control. 
The lack of adequate social support is one of the factors that increase the burden placed on primary caregivers (Prieto et al., 2017). In short, caregivers of ALS/FTD patients face a number of challenges that may well stretch their emotional and psychosocial abilities to the limit.

The main aim of this guide is to provide useful information on the disease, highlighting the potential evolution of patients from the early stages when the first symptoms appear through the disease process to the end of life.

Dementia has a high social impact on both sufferers and their social and family surroundings. Data from Spain's National Statistics Institute (INE) indicates that prevalence among the over 65 s is between $5.2 \%$ and $16.3 \%$, though it reaches $22 \%$ among men aged over 85 and $30 \%$ among women in the same age range. Dementia appears in 15\% of ALS sufferers, generally around the age of 60 (Lillo, Savage, Mioshi, Kiernan \& Hodges, 2012).

There is considerable evidence to suggest that $10-15 \%$ of ALS patients have frontotemporal dementia (FTD), characterised by personality changes, irritability and impairment of thinking and language functions. This has now come to be considered as a distinct variant of ALS, known as ALS/FTD (Strong, Abrahams, Goldstein, Woolley, Mclaughlin, Snowden \& Rosenfeld, 2017). The proportion of patients who have this variant and the factors and genes involved are currently being studied. For the moment, the data seem to indicate that the treatments recommended for ALS work less well in ALS/FTD patients, whose survival time is lower than that of sufferers without dementia (Govaarts, Beeldman, Kampelmacher, van Tol, van den Berg, van der Kooi \& de Haan, 2016).

The main behavioural disorder associated with ALS/FTD is apathy, followed by a loss of inhibition. In these cases patients are unaware of the changes that they are undergoing. Patients also frequently show cognitive disorders of the type known as dysexecutive syndrome, involving the inability to organise resources or envisage the consequence of actions, and language impairment (Murphy, Factor-Litvak, Goetz, Lomen-Hoerth, Nagy, Hupf \& Bedlack, 2016; Strong, 2008).

ALS is a serious disease whose course and evolution require the presence of caregivers to help tackle the challenges of providing assistance in a wide range of day-to-day activities in the wake of the deterioration of the patient's motor functions.

The need for care increases as the disease progresses and eventually full-time care is required. 


\section{MND}

\section{Motor Neurone Disease}

A neuromuscular degenerative disease

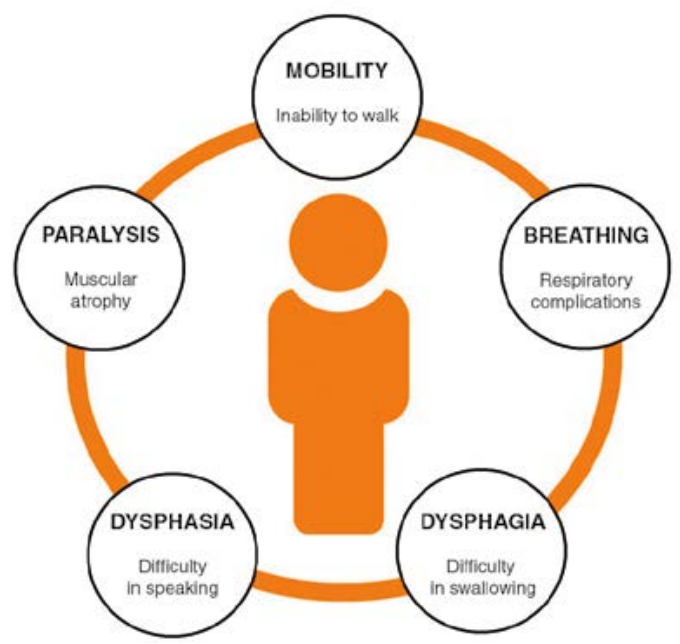

Facts and Figures on MND

- The average age at which symptoms appear is 60.5 years.

- $5-10 \%$ of cases are family forms, inherited mainly in dominant autosomal fashion.

- Men are more prone to it than women, by a ratio of 1.78:1.

- In Spain its prevalence is 3.5 cases per 100000 head of population. This means that there are around 4000 cases.

The disease may appear before age 40 or after age 70 , so the person who takes on the role of caregiver may be someone with whom the patient lives, i.e. a parent, child or sibling. Life expectancy among ALS sufferers is low, so the emotional burden on the immediate

These alterations in behaviour associated with cognitive deterioration have a notable impact on the burden placed on caregivers family is immense, and is only exacerbated by the high level of physical dependency of the patient and by a further variable that may come into play: behavioural disorders. Between $10 \%$ and $20 \%$ of ALS sufferers experience impaired judgment, personality 
and behavioural disorders that meet the clinical criteria for consideration as dementia.

\section{ALS/FTD: Main characteristics}

\subsection{Diagnosis-related problems: early symptoms and associated difficulties}

The most common scenario is that of a family member who expresses concern because an ALS sufferer is experiencing mood swings or language problems. As soon as these changes begin to manifest, it is advisable to inform the patient's family doctor so that a number of contingency measures can be set in motion to adapt care activities and prepare the patient's home. The aim is to prevent falls and other domestic accidents.

Clinical assessment usually entails separate assessments of the person affected and the main caregiver. This enables family members to raise honest concerns about how patients are functioning in their day-to-day activities and relationships, and helps to build up a comprehensive record of the appearance and duration of problems. This process also helps to determine whether there are any differences of opinion between the person suspected of having dementia, their family, friends and others close to them in regard to what is occurring, and whether there are any social or ambient circumstances that could provide a more likely alternative explanation. Thus, private interviews with patients usually cover habitual and

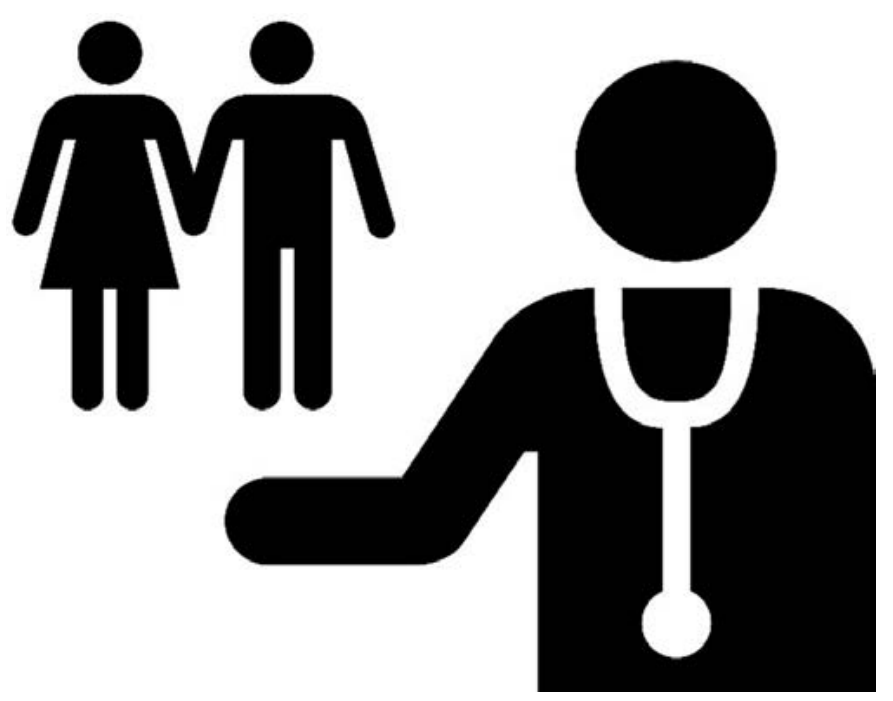


treatable causes of cognitive impairment, states of anxiety and depression and other causes, including the possible use of certain medicaments and the coexistence of other psychiatric disorders.

Paying attention to the progress of symptoms in a loved-one can be a complicated matter: it is only natural to deny such changes or struggle against them. However, denying or seeking to correct behaviour in inappropriate ways can harm relationships and care. This is why an objective assessment is needed, so help the caregiver and reference personnel to establish guidelines in day-to-day activities.

\subsection{Legal and financial issues}

Once dementia is detected in an ALS sufferer three actions need to be taken to safeguard the patient and those around him/her from potential legal complications as the deterioration produced by the disease progresses, e.g. in matters of wills, administration of property, etc. This is the time to seek legal advice on the granting of powers of attorney, guardianship and declarations of mental incompetence, as outlined below.

By granting powers of attorney sufferers can designate someone to care for them and hold decision-making powers over their future and over their goods after their death. This measure is intended to prevent future family problems and ensure the financial welfare and upkeep of the patient.

Guardianship entails the appointing of a legal guardian to manage the assets of the sufferer. This procedure is used only in acts in which sufferers cannot act for themselves. In the case of persons declared mentally incompetent, its purpose is to ensure the assistance of the guardian in acts expressly indicated by the relevant court ruling.

Finally there is the declaration of mental incompetence. This is a court procedure by which a tutor is appointed (with the tutor under the supervision of a judge and a public prosecutor as regards financial activities). The tutor is obliged to submit annual accounts. The tax authorities in Spain also provide aids, which vary from one regional autonomous community to another, to help persons with certified disability levels of $33 \%$ or more. These tax benefits may include one or more of the following: a) reductions in personal income tax; b) reductions in VAT in income tax returns; c) exemption from various traffic taxes; $d$ ) rebates on capital gains tax and on payments for inheritances and donations. These benefits are applied directly to sufferers, guardians or legal representatives. 


\subsection{Communication with sufferers, with children and with others}

The onset of behavioural symptoms such as apathy means that changes are needed in the way in which others communicate with sufferers. Irritability, agitation and apathy can be serious barriers that undermine necessary day-to-day communication. If there are children in the household then strategies also need to be set in place to improve the conditions of everyone involved.

\subsubsection{Communication with the patient}

It is important to be careful how one addresses the patient. The right words, tone of voice and body language and the healthy use of humor can help in dayto-day contact. Any temptation to treat sufferers as if they were children or have lost their sense of self must be avoided. Respectfully and thankfully recalling the care received in the past from sufferers can provide the motivation needed to offer them the attention and support that they need day by day. Sometimes language and the way in which patients can communicate may be affected due to dysarthria, i.e. problems in articulating speech.

Changes in behaviour among patients may result in outbursts of anger and arguments with those close to them. The first step towards avoiding arguments with patients is detachment. This means cultivating emotional indifference or

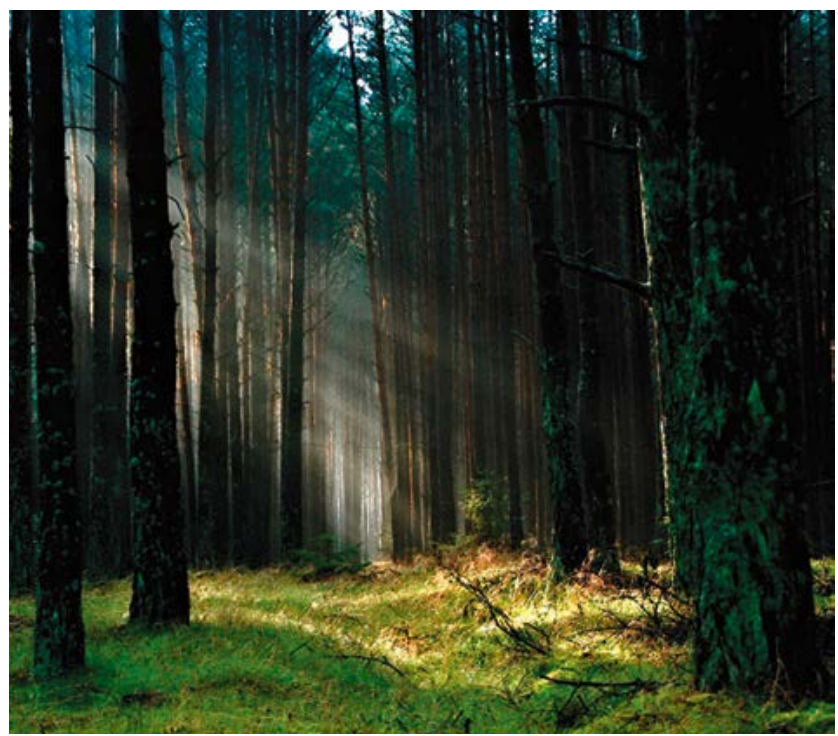


acting as if there is no problem. Detachment means genuinely accepting the other person as they are rather than as one would like them to be.

When you take on board that a loved-one is suffering from a disease that affects their ability to think, to react and to understand the world in the way that you do, you can begin to understand the change that has taken place. This enables you to cope with the situation in a less personal way and respond more effectively to the challenges posed by caring for an ALS/FTD sufferer. Such detachment can only be attained effectively by taking great care and by listening to the patient with respect. This means holding back emotional reactions long enough for the patient to feel listened to, and detecting what is going on in the patient's interior. For example if the patient suffers a burst of anger when told to take their medicine it may be helpful to ask yourself "How would I feel if this was me?" The patient may be tired of always being told what to do, or may be upset by the tone of voice used or the way in which a question was asked. They may be irritated because they were interrupted during a regular routine, or because they do not really understand why they have to take the medicine in question.

In this context, detachment means finding out what emotions are concealed beneath the words and actions of the patient and learning to respond to them calmly and with ease rather than taking things personally.

When someone is upset or agitated, redirecting their attention away from the situation that provoked the reaction can be a powerful tool. The first step in distracting them is to help them do something that they find pleasant (e.g. putting on music that they are familiar with, a video that they like, etc.). This may help them to relax and thus distance them from whatever disturbed them. Another idea is to ask them to help in simple tasks, if motor function permit it. Distracting them may mean selectively ignoring things, because helping them to focus on something pleasant may mean ceasing to pay attention to what they are doing at that time. This can be done by ignoring their complaints and changing the subject to a more neutral, more pleasant topic, e.g. the weather outside or other strategies as the case may be.

At first using this type of communication tool may feel awkward: it may seem childish or rude to ignore certain comments and answer with a non sequitur. But ignoring or distracting someone to help them not to feel frustrated and not to behave aggressively or in an agitated fashion is not rude. Indeed, in this the caregiver is providing patterns that help the sufferer.

In the course of the disease it is common for changes to take place in the social environment of sufferers, and for some people to distance themselves. For various reasons, ALS/FTD sufferers sometimes object strongly to receiving certain types 
of help from specific individuals. They may even become hostile to their main caregiver and come to favor other people in their surrounding environment. In such cases the path of least resistance may be to take advantage of everyday circumstances such as personal hygiene and the administration of medication. Some advice on communicating with dementia sufferers is given below:

\begin{tabular}{ll} 
Patience & $\begin{array}{l}\text { Take things slowly. } \\
\text { Do not ask more than one question at a time. } \\
\text { Give the patient a moment before repeating instructions. } \\
\text { Speak slowly and clearly, without raising your voice. }\end{array}$ \\
\hline Divide tasks into smaller sub-tasks. \\
Use hand gestures to refer to objects in particular. \\
Use written notes and clarifications if you find them helpful. \\
Bear in mind how the patient may feel in situations of agitation or apathy.
\end{tabular}

\subsubsection{Communication with children}

Sometimes dementia may occur at an age when they have children. If so, those children will be affected by the process, as they are a highly vulnerable group. Caring as a child for a parent with ALS/FTD implies a role reversal between parents and children. As the capabilities of the patient decrease, children may increasingly take on the role of caregivers. It is important to be sensitive to the needs of children at this time and to allow them an outlet for expressing their feelings.

As the disease progresses and the patient's inhibitions are lost, there may be behaviour liable to cause shame or other negative feelings in children. In such cases children may turn their anger and frustration onto the main caregiver. If this happens, it is advisable to seek external help in dealing with the range of emotions entailed in having a parent with ALS/FTD. 
If school-age children are involved, it is advisable to notify teachers, authorities and others of the situation in the home so that they are aware of the personal circumstances of the children. At the same time, problems may arise at school for various reasons. Children who are undergoing these upsets in their families at home may question the priorities and importance placed by adults on academic performance, school work and attendance. To forestall this, it is important for them to maintain a life separate from the reality of the disease. Having someone close to the family to talk to may give children the freedom to express frustrations that they cannot share with close relatives who are also suffering them.

\subsubsection{Talking to others about the disease}

Each case is different, depending on family dynamics, personalities, etc., but in general it is advisable to tell children as much as possible about the reality of the situation. Caregivers should not in any event feel under any pressure either to disclose or to conceal their domestic situation: they should disclose whatever information they wish and hold back as much as they want.

The disease process often leads to readjustments in communication with others and puts a new perspective on the desire to talk to others about the condition of the patient and how the caregiver is experiencing the process. Sometimes caregivers may be reluctant to share their feelings for fear of condescending reactions, out of a desire not to give an impression of vulnerability or for reasons of discretion. As the disease progresses, caregivers shape and adapt their attitudes until they find the formula that best suits both them and the patient.

In general, when the truth is shared the reactions of others tend to be better than expected.

\subsection{The caregiver: the hub of the care system of the patient}

How can one relate to a person who is physically dependent and feels no empathy? Each case, and each patient, is different and may be difficult and stressful. Caring for someone who has ALS/FTD entails challenges, such as the following:

1. Uncertainty as to the rate at which the disease will progress, i.e. whether motor functions will be lost quickly or slowly.

2. The short life expectancy of persons diagnosed as having ALS. 
3. The unpredictability of patients' behaviour and the problems entailed by a lack of inhibitions, compulsive, aggressive behaviour and speech difficulties.

4. The lack of empathy and emotion shown by patients, which undermines the strength of caregivers and may lead them to feel demoralised, neglected and irrelevant.

These factors mean that caregivers of ALS/FTD patients are at considerable risk of suffering depression, bad moods, sleeplessness and chronic anxiety. It must also be remembered that it is painful to care for someone whose range of emotions may be compromised.

In most cases, care is given by informal caregivers, mainly family members and close friends, who provide it on an altruistic basis for individuals who have some degree of disability or dependence.

In some cases patients may shift from normality to complete dependence in a short time, so that they require specific care. This is usually provided by members of their immediate family: parents, spouses and children. Providing this care is demanding and entails a high level of responsibility, which may give rise to stress and emotional upsets among caregivers and other family members. This may have serious consequences for their physical and mental health if the burden of care becomes too great, such as depression and psychosomatic disorders. Caring is a complex process that features a blend of

If caregivers have sufficient

outside resources and coping mechanisms to help them adapt

their role does not tend to be frustrating and may actually have rewarding aspects many emotions, such as guilt, shame, rage, sadness, confusion and impotence. All these emotions may show up at different times and to varying extents. However, experiencing such feelings does not make people bad caregivers: the emotions involved produce stress and overlay more positive emotions that may arise during the process.

Caregivers striving to provide the best care possible often neglect to take their own best interests and dreams into account. It is not unusual for caregivers, seeking to respond to all the challenges that crop up during the disease, to lose their own personal identities and become mere extensions of the person cared for, thus running the risk of falling ill themselves.

The mechanisms available for dealing with the problems that arise in the course of the process include coping techniques: coping based on emotion is closely linked with health problems (it negatively affects or modifies the assessment of the situation) but coping focused more on the problem itself is associated with a reduction in stress.

Each case is unique, but some reactions are shared by all caregivers, such as impatience, irritation and tiredness. These attitudes are often hard for caregivers 
to admit to themselves or to others. The problem does not lie in the appearance of these reactions but in how to deal with them.

\subsection{Accepting the disease: resilience}

People who are trapped by their feelings are slaves to the reactions that those feelings provoke. The ALS/FTD process leaves patients incapable of helping caregivers make certain decisions. This is painful for people who are accustomed to sharing important events in their lives. They have to accept that someone whom they know intimately is incapable of running their own life. Caregivers sometimes feel that they are being dishonest or disrespectful on watching their words when they speak to their loved ones.

Acceptance of the disease is a critical aspect for resilient caregivers.

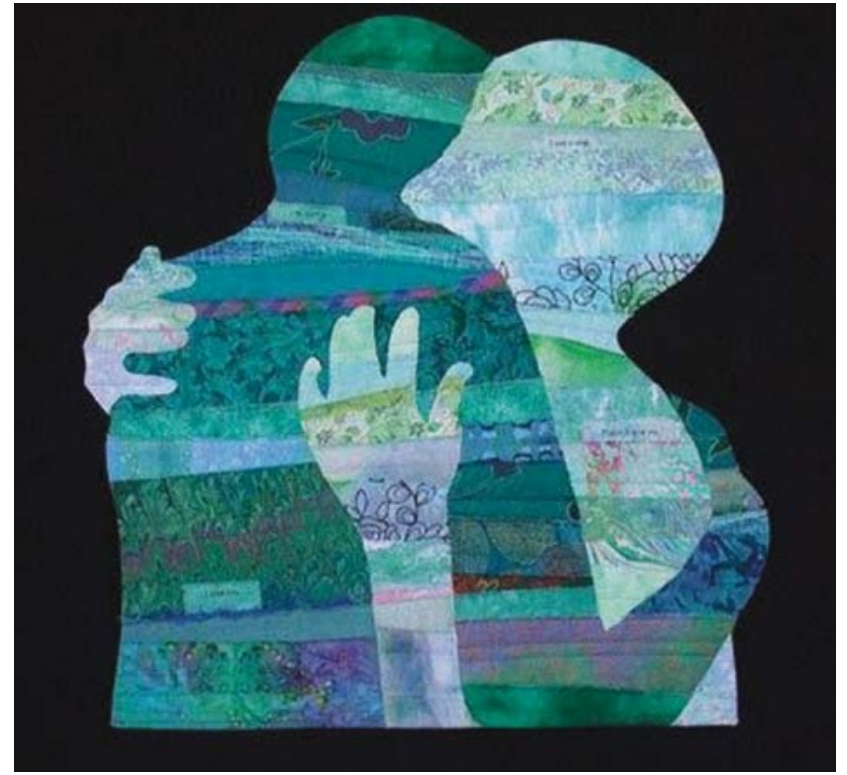

It may take time to set realistic expectations, which may in any event need to be readjusted at intervals as the ALS/FTD progresses. Accepting limitations also means letting go: accepting your own internal reactions to dementia is the most 
complex, difficult challenge of all. Caregivers who set out to perform their role perfectly may become demoralised when they fail to live up to their own personal, idealised expectations. In extreme cases they may feel a desire to "let the patient go", given the lack of support and absence of a cure for the disease. This feeling is commonplace and may arise at any time during the process. Caregivers need to accept it and be aware of it, in spite of the guilt associated with it.

On a day-to-day level, failing to accept the difficulties entailed by caring may lead to an unnecessary delay in requesting help when necessary. Acceptance means taking on board the ups and downs that are bound to appear: however much experience a caregiver may have of ALS/FTD sufferers there is always more to be learned. However, resilient caregivers learned to maintain a healthy review of care and to trust that their own values and fundamental principles will guide them, whatever challenges they may face they by day.

The inability to accept the reality of ALS/FTD may undermine even the best attempts to avoid arguments and may hinder effective communication. Caregivers who use resilience understand thor-

Acceptance is a necessary

first step in setting up resilience mechanisms oughly how the disease affects the persons cared for, and hold realistic expectations regarding their loved-ones and themselves. Setting up and mastering these mechanisms is a complex learning process.

Another issue concerned with acceptance is taking on board the limitations that caregivers and their loved ones may experience. When motor functions are not too seriously affected, many of the problems experienced by ALS/FTD sufferers may be passed over, and it is sometimes easy to believe that they are still able to do all the things that they could do previously. Once the diagnosis is accepted, caregivers may realise that they are expecting or asking their loved ones to do things that are no longer possible for them. Caregivers then have to begin to do things differently. Acceptance of these new limits also means forming expectations that are realistic for the caregiver and for others who helped during the process. Accordingly, this is the time for caregivers to ask themselves if it is necessary or reasonable to seek outside help. They need to look around and assess their own support systems realistically. They will probably find that there are people around them who can support them and who are only too willing to help in the process, though others may distance themselves from the patient and their family.

Acceptance means learning to recognise thoughts, feelings, memories and reactions, i.e. seeing things the way that they really are. Seeing that a loved one can no longer do things that used to come easy to them can be painful and hard to accept. Indeed, caregivers often feel that accepting that a loved one has an incurable neurodegenerative disease is tantamount to abandoning them. They 


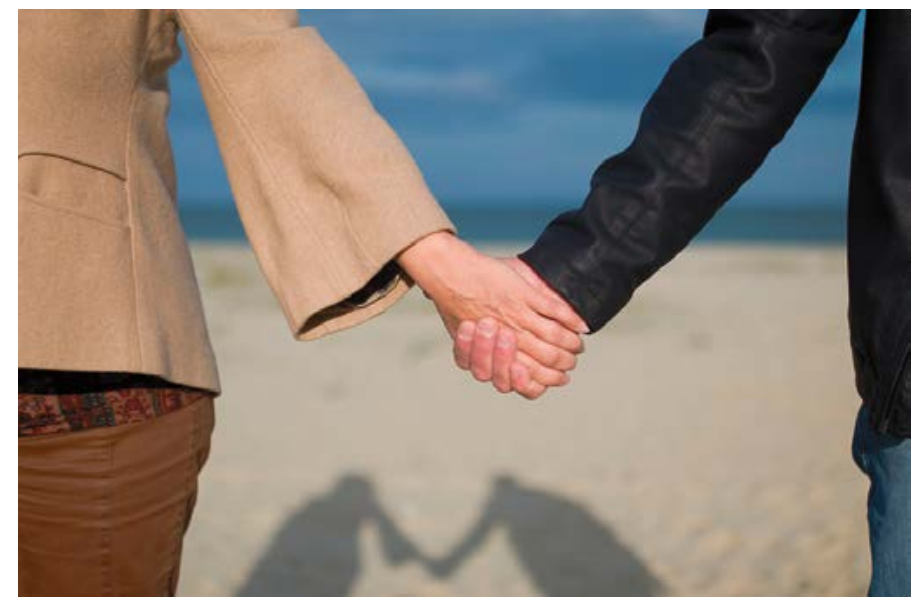

want the patient to keep fighting, and seek to delay or reverse the progress of the disease. They may become angry when they failed to achieve this, and may later feel guilty at having become angry at their failure or at having become annoyed with someone who is not responsible for their behaviour. Feelings of guilt are commonplace. They are the reflection of tough emotional demands, but may cause caregivers to feel ashamed and frustrated during the process.

Even caregivers who have fully accepted the fact that their loved ones have MND find it hard to accept the onset of severe behavioural disorders.

When ALS sufferers develop dementia, the resulting cognitive and emotional changes tend to take place gradually, and they insist that they are all right. ALS sufferers may have behavioural problems that have developed over just a few weeks. Caregivers may assume that these changes are the result of the patient feeling irritated or sad, or going through a bad time, or may even believe that they are doing things simply to annoy their caregivers if they have shown similar behaviour in the past. The same goes for cases in which patients have previously suffered from depression.

It is important to realise that their current behaviour is not caused by the same reasons as in these past experiences. People with dementia are less and less able to understand properly the consequences of their actions, and cannot see how much help they need from others to perform certain tasks. They find it hard to finish what they have started, to understand the emotions that they spark in others and to control their rapid changes of mood and attitude and their states 
of agitation. Realising this may not prevent caregivers from feeling angry or disappointed, but they will gradually come to understand that these attitudes are part of the disease and this in turn will help them tackle things differently.

\subsection{Respite care for caregivers}

Studies of caregivers of ALS/FTD sufferers suggest that the earlier they take their own needs for social support and respite into account, the better their own

In this context, the term means

short periods of rest or relief
What is respite?

quality-of-life and that of the patient becomes (ed Wit, Bakker, van Groenestijn, van den Berg, Schröder, Visser-Meily \& Beelen, 2017). All caregivers need to find suitable space for respite.

Each caregiver has different habits, tastes and interests, so what constitutes respite for one may not do so for another. Some ideas for respite include taking a walk, visiting friends, going for a coffee, reading the newspaper, doing some exercise, meditating, dancing, going out for lunch or watching a film with friends.

It is important to know what to do during respite time, and to realise when respite is necessary. Ideally, this should be thought out and set in place before a crisis occurs. At first, stress levels are low and the persistent messages that they send out are often denied or ignored. One way of determining whether a caregiver is under chronic stress is to watch for symptoms such as headaches and backache, upset stomachs and high blood pressure. These may be the result of poorly handled stress.

Symptoms of depression may include sleeplessness, lack of appetite, listlessness and a feeling that you would "rather be anywhere else but here". Clinical manifestations of anxiety include inability to concentrate and panic attacks, characterised by a racing heartbeat and laboured breathing. Caregivers frequently fail to realise when they are bordering on exhaustion, though patients may notice, as the standard of care may suffer: they know that the caregiver is emotionally elsewhere.

In matters of care, as in many other aspects of life, quality is more important than quantity. Care must not be driven by rage, guilt or resentment.

There may be various reasons why caregivers fail to ask for help when they need it. In some cases they may wish to 
protect the feelings and the dignity of the patient: they are reluctant to ask family or friends for help because they do not want them to see a loved one suffering from a neurodegenerative disease. In other cases they prefer people to remember patients as they were in better times. Moreover, caregivers often do not want to feel the compassion or shame expressed by third parties who may not understand the situation. Caregivers may also wish to protect others who have their own jobs, leisure pursuits and obligations, and may be reluctant to request help because they do not wish to place responsibility on those others.

They wrongly fear that if they ask for help it may be denied or given only reluctantly, which they feel may be worse than not asking. Finally, if caregivers depend on the goodness of others and have to request help in tasks other than caring for the patient, they may use this as an excuse to refuse or fail to carry out those tasks.

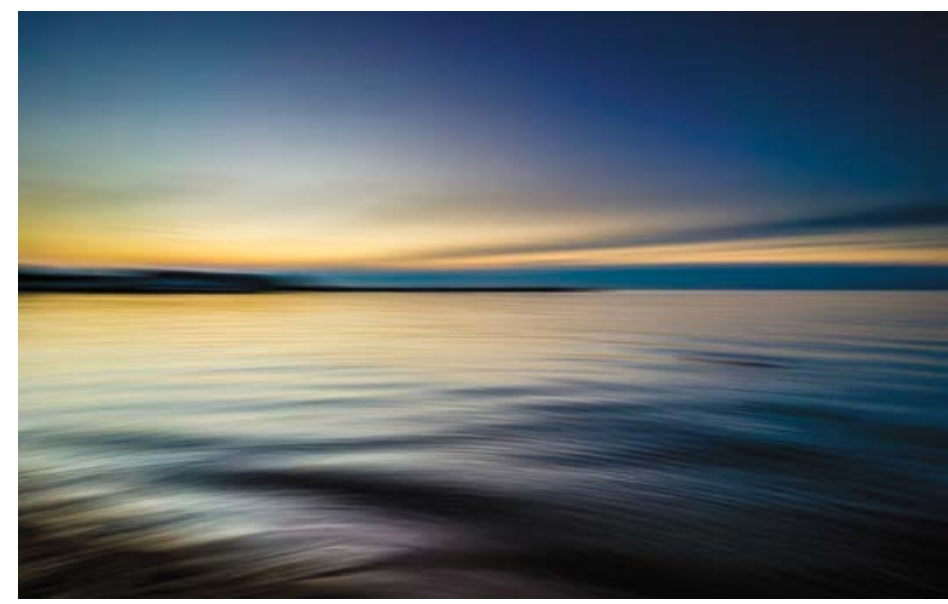

Neglecting their own physical, emotional and spiritual health may harm caregivers. Initially, not taking care of their own health does not seem to make much of a difference: they may just feel a little more tired than usual. They may start to neglect formerly frequent pastimes such as taking exercise or visiting relatives. Caregivers may gain or lose weight, or may suffer high blood pressure, without feeling ill enough actually to see a doctor. In many cases caregivers may also have to work outside the home, and over time prolonged stress can undermine their physical and emotional health (Kasuya, Polgar-Bailey \& Robbyn Takeuchi, 2000; Krishnan, York, Backus \& Heyn, 2017; Ybema, Kuijer, Hagedoorn \& Buunk, 2002).

Family conflicts may arise out of the management and organisation of care for the patient. These problems can substantially undermine the morale of the main 
carer, so it is advisable to set up caring routines as soon as possible among all those involved in the task, from the personal hygiene of the patient to days or times of respite.

Once caregivers consciously decide to begin to take care of themselves the first challenge that they face is to find time to do so. This is something that the all need to do this for themselves: it does not need to be much time, but it must be often and at regular intervals. Time is a valuable asset for everyone, but this is particularly so for caregivers of patients suffering from neurodegenerative diseases.

\subsection{Asking others for help}

Caregivers need to remember that caring for a loved one is no substitute for the need to regularly do something that they enjoy or that inspires them and fills them with energy. Doing something that you love every day helps you to stay in contact with your creative, curious side, which makes life meaningful. If they wish to become resilient, caregivers need to cultivate these aspects.

Formal caring services such as adult health programmes and care in the home have helped millions of caregivers and their loved ones. However, not everyone wishes to use them, or indeed is able to do so. That is why respite care for caregivers may take the form of help from family and friends. Indeed, even those caregivers who do use formal services almost always need different types of care other than those provided by health services and formal caregivers. Patients and caregivers have people in their lives who love them and are willing to help, but simply do not know how to.

On the other hand, caregivers often do not know what help to ask for from others. When they ask someone else for help, they have to face the possibility that the answer may be no. They may also feel criticised for not being capable of handling the situation unaided. In such cases, caregivers may not know what to say to people that they do not know well or with whom they lack the necessary trust; and they may find it more difficult than it seems to ask for help from those closest to them. The other main reason why caregivers fail to ask for help is that they simply do not know what type of help they really need or who they should ask for it.

One way of finding out what type of help is needed is to make a list. For instance caregivers can ask themselves the following: What is a normal day for me? Do I need help in preparing food for the patient, helping them eat, get dressed or shower? Are there any errands that need doing but I don't have time to go to the shops? What type of jobs need doing in the home? Thinking about these points can bring to light areas where occasional help from others is needed, e.g. in housework or seasonal tasks such as Spring cleaning. Other points may be more personal, but just as necessary, such as taking a few hours off every Saturday morning. 
Over time caregivers may come to feel that these things are not important, or that the time that it takes to explain what needs to be done or the possibility that the help may need to be repaid in the future mean that it is not worthwhile asking for it. Some caregivers lack the clarity of thought required to recognise needs as they arise, and may also not be sure who to ask for each type of help. A good way of determining needs is to make a list of the people with whom you are in touch day by day. The typical list includes family, neighbours, friends, workmates and other members of service organisations, though some people are likely to have distanced themselves as the disease has progressed. Now take the list of activities with which you need help and decide which ones could be delegated to someone else. Compare the two lists, and you are sure to find some people better suited to some tasks and some to others. In some cases there is no need for a high level of trust (e.g. to ask someone to go to the chemists for you), but in others a closer relationship may be required (e.g. to ask someone to stay with the patient while you take a morning off).

\subsection{Psychoeducation of caregivers}

Psychoeducation is an educational method that seeks to provide quality information on various aspects of a given disease, such as its symptoms, expected course, consequences and prognosis. It also serves to dispel myths and misconceptions, to highlight the treatment options available and to help identify signs of potential worsening of the disease. Psychoeducation is a process that seeks to improve the skills of caregivers by providing an information flow that runs both ways rather than just one way.

People who undergo this process learn more about various aspects of the disease, and learn to identify signs or symptoms indicative of anxiety and depression that may proceed a crisis. Providing information on the disease forms part of numerous intervention programmes. It is important for clinics and associations to give caregivers sufficient information on the symptoms of ALS/FTD to enable them to identify changes in their physical or mental state and anticipate any potential worsening or crisis, thus lessening the impact of the disease on their own lives.

\subsection{Results of studies involving ALSIFTD caregivers}

Caring for patients with ALS/FTD can be too much of a burden, given the symptoms and problems associated with the disease. As a result, research has been conducted into what types of intervention are most suitable for reducing the burden on caregivers and what characteristics define the best adapted caregivers. 
Cognitive behavioural therapy is one of the techniques that has proved useful in improving the quality-of-life of informal caregivers. This therapy is based on explaining that symptoms and behavioural disorders arise from the thinking process. Seeking to change beliefs and dysfunctional thinking can help to reduce psychological symptoms. A study by Van Froenestikn et al. (2015) shows that quality of life among caregivers of ALS patients increases after such interventions, and that there is a moderate reduction in the feeling of being overburdened.

Dignity therapy was developed to reduce psychosocial and existential stress arising from the loss of a loved one. It requires caregivers to recall aspects of their lives that they wish to hold onto, and helps them find a purpose and goal in life and to express themselves in the context of terminal illness. Studies such as Bentley, Aoun, O'Connor, Breen \& Chochinov (2012) highlight the importance of applying therapy of this type with the families and caregivers of ALS/FTD patients, as it seems to have a positive impact on their quality-of-life.

Meditation based on mindfulness has also

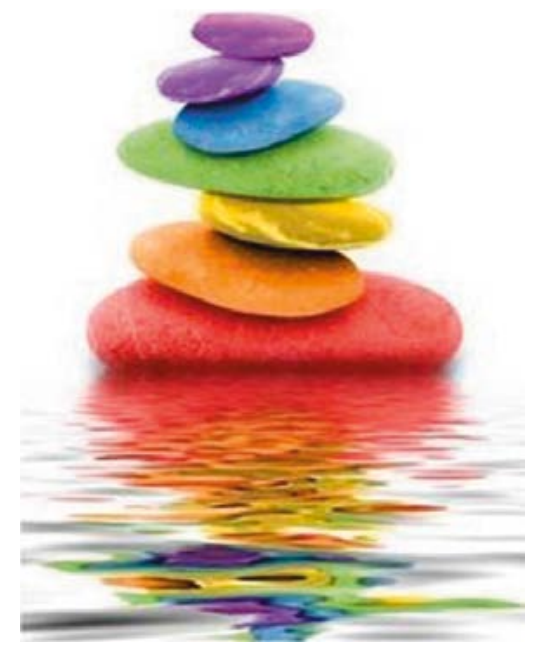
been tried with many chronic diseases and found to reduce stress in most cases. This technique is based on Buddhist meditation. Mindfulness means paying attention consciously to the present, without judging what is going on. This provides people with a greater awareness of what is happening to their bodies, and thus helps them achieve a better balance of emotions and greater emotional stability. This provides a sound basis for reducing stress and increasing resilience. The research group of Pagnini, Di Credico, Gatto, Fabiani, Rossi, Lunetta \& Banfi (2014) examined such interventions in ALS sufferers and found them to be a useful tool, especially during the early stages of the disease.

Support groups of caregivers have been found to be highly beneficial as effective psychological interventions to help caregivers of FTD sufferers. Such groups tend to work on educational content, providing information on medical, legal, financial and insurance matters and on resources and services available. One of their most important aspects is their therapeutic side, as they enable caregivers to share their experiences. This fosters mutual support, helps them express emotions and facilitates the development of coping strategies for handling the behavioural symptoms that go with the disease. Support groups are seen by caregivers as unnecessary aid that helps them reduce the burden of care (Nunnemann, Kurz, Leucht y Diehl-Schmid, 2012). 


\section{Conclusions}

The role of caregivers is fundamental, as they help to improve the health and emotional and social circumstances of patients. This task is particularly important in regard to ALS, given the state of complete dependence that sufferers eventually reach.

Behavioural, judgemental and cognitive disorders are not unusual and can be a tough challenge for those close to the individuals affected (spouses, children, parents and friends). The great internal and external burden and pressure on caregivers means that they tend not to realise that mistaken approaches in care can have repercussions on the relationship between care and patient, on caring tasks and indeed on their own health and surroundings.

The wide range of emotions that arise during the process often lead caregivers to deploy measures to cope with their feelings. It is entirely understandable and only to be expected that caregivers are not always able to handle these emotions well, given the great demands imposed by ALS/FTD. Failing to recognise the signs that the burden has become too great and that they are suffering from emotional wear and tear can lead caregivers to provide lower standards of care and can result in lower quality-of-life for both caregivers and patients.

This guide sets out strategies to help recognise these signs, and guidelines for helping to deal with them. The clinical and social reality of dementia in ALS is coming to the forefront in the process of care. This briefing is intended as an initial point of support for caregivers of ALS/FTD sufferers and as a basic reference document for medical personnel and associations.

\section{References}

Bentley, Brenda, Aoun, S. M., O'Connor, M., Breen, L. J. y Chochinov, H. M "Is dignity therapy feasible to enhance the end of life experience for people with motor neurone disease and their family caregivers?" BMC palliative care, 11.1(2012), 18. 18.

de Pedro-Cuesta, Jesús, Virués-Ortega, J., Vega, S., Seijo-Martínez, M., Saz, P., Rodríguez, F., ...\&Martínez-Martín, P. "Prevalence of dementia and major dementia subtypes in Spanish populations: a reanalysis of dementia prevalence surveys, 1990-2008." BMC neurology 9.1 (2009): 55.

de Wit, Jessica, Bakker, L. A., van Groenestijn, A. C., van den Berg, L. H., Schröder, C. D., Visser-Meily, J. M. \& Beelen, A. "Caregiver burden in amyotrophic lateral sclerosis: A systematic review." Palliative Medicine (2017): 0269216317709965.

Govaarts, Rosanne, Beeldman, E., Kampelmacher, M. J., van Tol, M. J., van den Berg, L. H., van der Kooi, A. J. \& de Haan, R. J. "The frontotemporal syndrome of ALS is associated with poor survival." Journal of neurology 263.12 (2016): 2476-2483. 
Kasuya, Richard T., Polgar-Bailey, M. P. \& MPH Robbyn Takeuchi, M.. "Caregiver burden and burnout a guide for primary care physicians." Postgraduate Medicine 108.7 (2000): 119. Krishnan, Shilpa, York, M. K., Backus, D. \& Heyn, P. C. "Coping With Caregiver Burnout When Caring for a Person With Neurodegenerative Disease: A Guide for Caregivers." Archives of physical medicine and rehabilitation98.4 (2017): 805-807.

Lillo, Patricia, Savage, S., Mioshi, E., Kiernan, M. C. \& Hodges, J. R "Amyotrophic lateral sclerosis and frontotemporal dementia: a behavioural and cognitive continuum." Amyotrophic Lateral Sclerosis 13.1 (2012): 102-109.

Murphy, Jennifer, Factor-Litvak, P., Goetz, R., Lomen-Hoerth, C., Nagy, P. L., Hupf, J. \& Bedlack, R. S "Cognitive-behavioural screening reveals prevalent impairment in a large multicenter ALS cohort." Neurology 86.9 (2016): 813-820.

Nunnemann, Sabine, Kurz, A., Leucht, S. \& Diehl-Schmid, J. "Caregivers of patients with frontotemporal lobar degeneration: a review of burden, problems, needs, and interventions." International Psychogeriatrics 24.9 (2012): 1368-1386.

Pagnini, Francesco, Di Credico, C., Gatto, R., Fabiani, V., Rossi, G., Lunetta, C. \& Banfi, P "Meditation training for people with amyotrophic lateral sclerosis and their caregivers." The Journal of Alternative and Complementary Medicine 20.4 (2014): 272-275.

Prieto, C., Eimil, M., López de Silanes, C., Llanero, M. \& Villarejo, A. (2017). Ver Informe de la Fundación del Cerebro. Impacto Social de la Enfermedad de Alzheimer y otras Demencias, Actualización. http://www.fundaciondelcerebro.es/docs/IS_EA_y_otras_ demencias.pdf (consultada el 12 de noviembre de 2017).

Strong, Michael J. "The syndromes of frontotemporal dysfunction in amyotrophic lateral sclerosis." Amyotrophic Lateral Sclerosis9.6 (2008): 323-338.

Strong, Michael J., Abrahams, S., Goldstein, L. H., Woolley, S., Mclaughlin, P., Snowden, J. \& Rosenfeld, J. "Amyotrophic lateral sclerosis-frontotemporal spectrum disorder (ALSFTSD): Revised diagnostic criteria." Amyotrophic Lateral Sclerosis and Frontotemporal Degeneration 18.3-4 (2017): 153-174.

Van Groenestijn, A. C., Schröder, C. D., Visser-Meily, J. M., Reenen, E. T. K. V., Veldink, J. H., \& Van Den Berg, L. H. (2015). Cognitive behavioural therapy and quality of life in psychologically distressed patients with amyotrophic lateral sclerosis and their caregivers: Results of a prematurely stopped randomized controlled trial. Amyotrophic Lateral Sclerosis and Frontotemporal Degeneration, 16(5-6), 309-315.

Wilson, C. M., Grace, G. M., Munoz, D. G., He, B. P. \& Strong, M. J. "Cognitive impairment in sporadic ALS A pathologic continuum underlying a multisystem disorder." Neurology 57.4 (2001): 651-657.

Ybema, Jan F., Kuijer, R. G., Hagedoorn, M. \& Buunk, B. P. "Caregiver burnout among intimate partners of patients with a severe illness: An equity perspective." Personal Relationships 9.1 (2002): 73-88. 


\section{Biographical notes}

DEUSTO Neuro-e-Motion research team, composed by Imanol Amayra (Director), Juan Francisco López Paz, Esther Lázaro, Oscar Martínez, Sarah Berrocoso, Maitane García, Mohammad Al-Rashaida \& Manuel Pérez, has been awarded on 14th February2018, the 1st prize of XIII edition of UD-Santander Research Awards for their research project on "Psychosocial evaluation and intervention of rare diseases". The project, led by the team Neuro-e-Motion, aims at developing evaluation and intervention strategies for improving the quality of life of people with rare diseases and their families, mostly through new technologies.

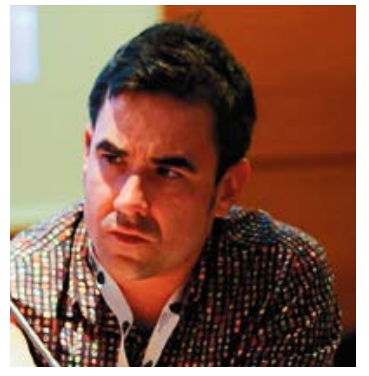

\section{Manuel Pérez Álvarez}

A neuropsychologist and researcher into cognitive disorders associated with MND. He holds a Master's Degree in Mental Health and Psychological Therapies from the University of Deusto.

\section{Oscar Martínez Gutiérrez}

Holder of a PhD in Psychology. A lecturer at the Faculty of Psychology of the University of Deusto. Head of the programme for the application of ICTs to research into neuro-muscular disorders.
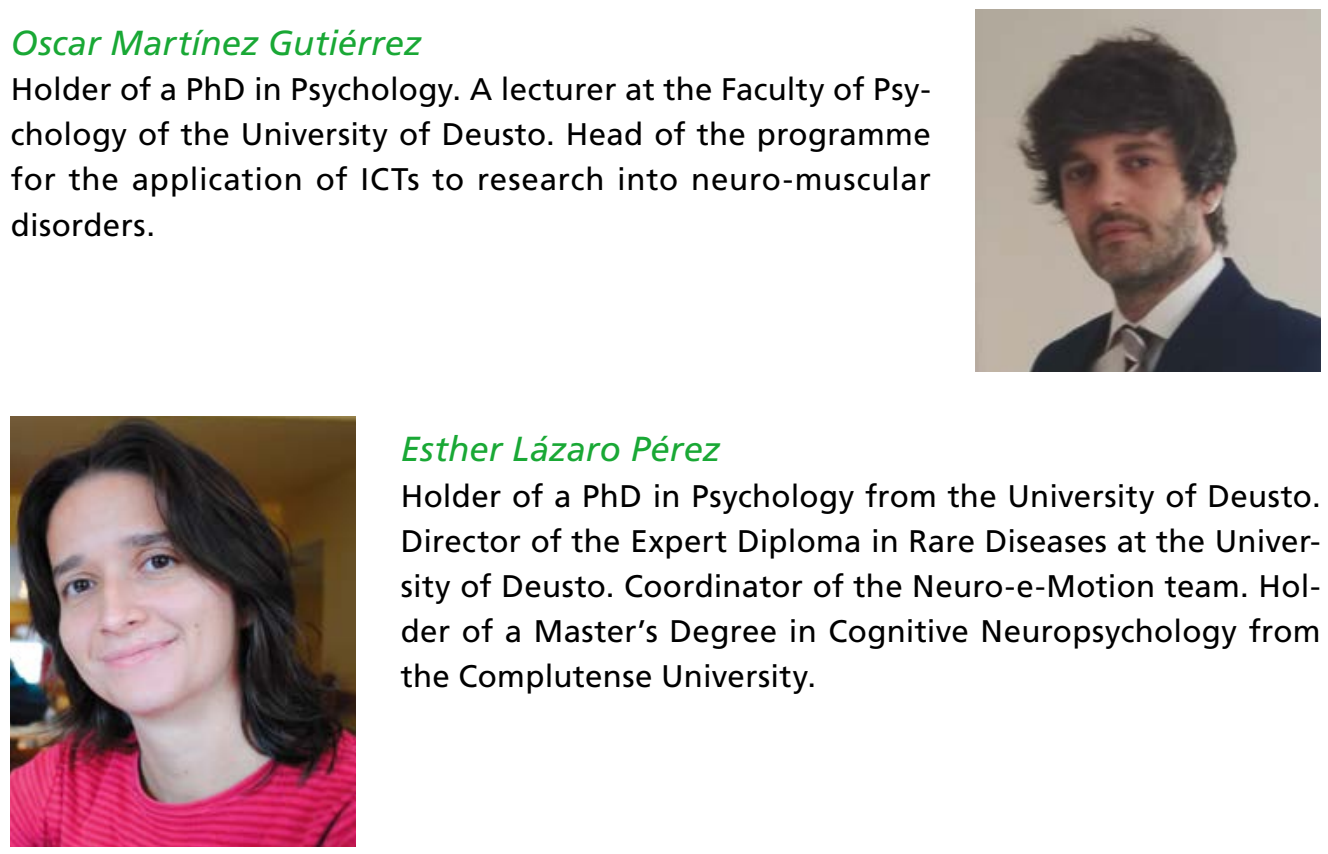

\section{Esther Lázaro Pérez}

Holder of a PhD in Psychology from the University of Deusto. Director of the Expert Diploma in Rare Diseases at the University of Deusto. Coordinator of the Neuro-e-Motion team. Holder of a Master's Degree in Cognitive Neuropsychology from the Complutense University. 


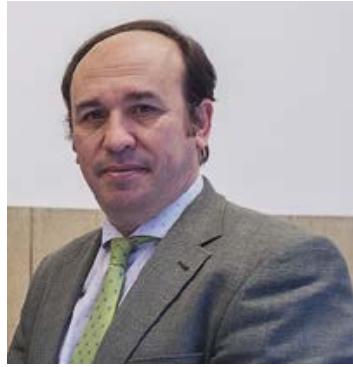

\section{Juan Francisco López-Paz}

Holder of a PhD in Psychology. A tenured lecturer at the Faculty of Psychology of the University of Deusto. Lecturer for the subject "Assessment and Clinical Intervention in Rare and Infrequent Diseases" at the University of Deusto.

\section{Alicia Aurora Rodríguez Bermejo}

Holder of the Chair of Psychology at the University of Deusto. Lead researcher on the Neuro-e-Motion team. Head of the Psychological Assessment, Personality and Treatment Department at the University of Deusto.

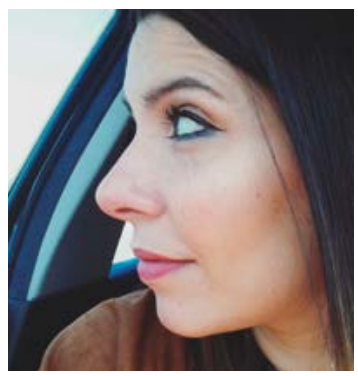

\section{Sarah Berrocoso Cascallana}

Researcher in remote assistance for carers of children with rare diseases. Holder of a Master's Degree in Mental Health and Psychological Therapies (Neuropsychology) and a Master's Degree in General Health Psychology from the University of Deusto.

\section{Maitane García Martín}

Researcher in Arnold Chiari malformation. Holder of a Master's Degree in Clinical Neuropsychology and a Master's Degree in General Health Psychology from the University of Deusto.
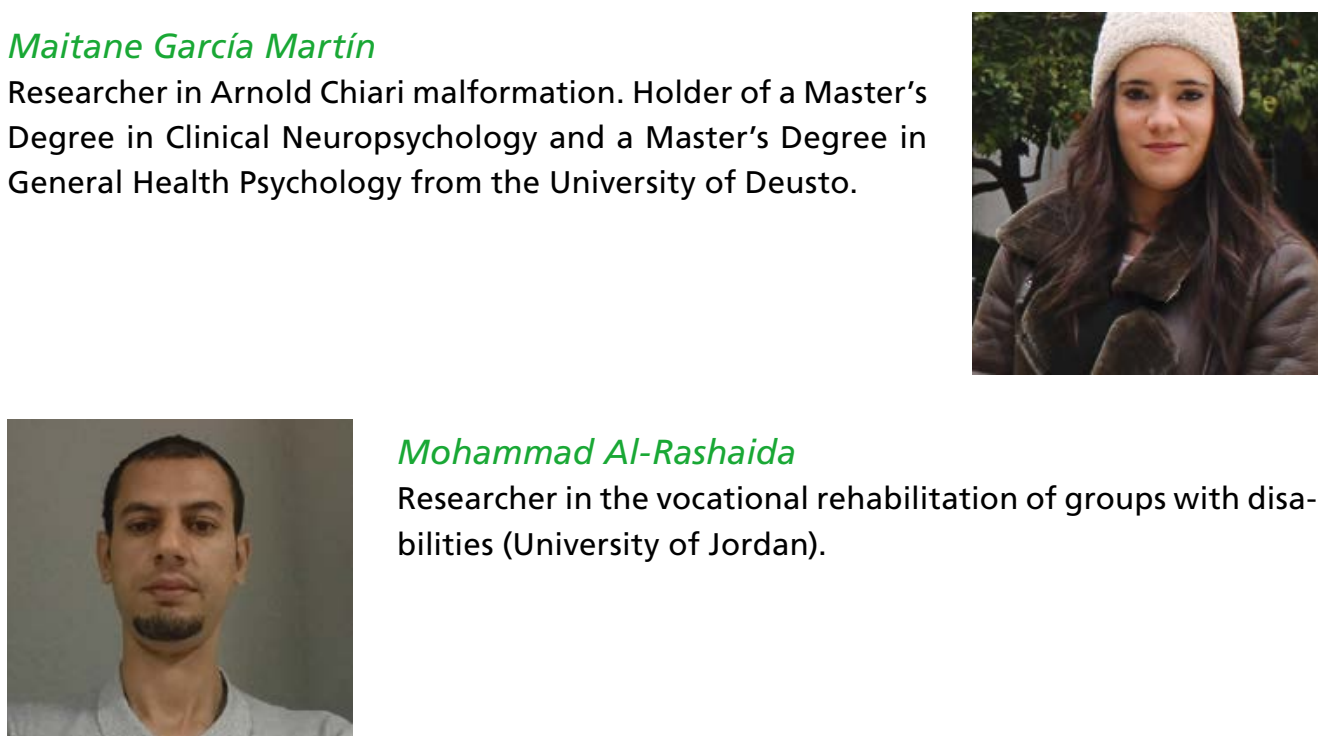

\section{Mohammad Al-Rashaida}

Researcher in the vocational rehabilitation of groups with disabilities (University of Jordan). 


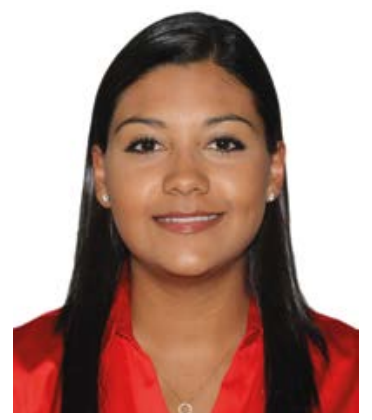

\section{Paula Luna Ovalle}

Clinical Psychologist (Francisco Marroquín University) Holder of a Master's Degree in Clinical Neuropsychology from the University of Deusto.

\section{Paula Fernández Elorriaga}

Researcher in new communication technologies for persons with dementia. Holder of a Master's Degree in Clinical Neuropsychology and a Master's Degree in General Health Psychology from the University of Deusto.
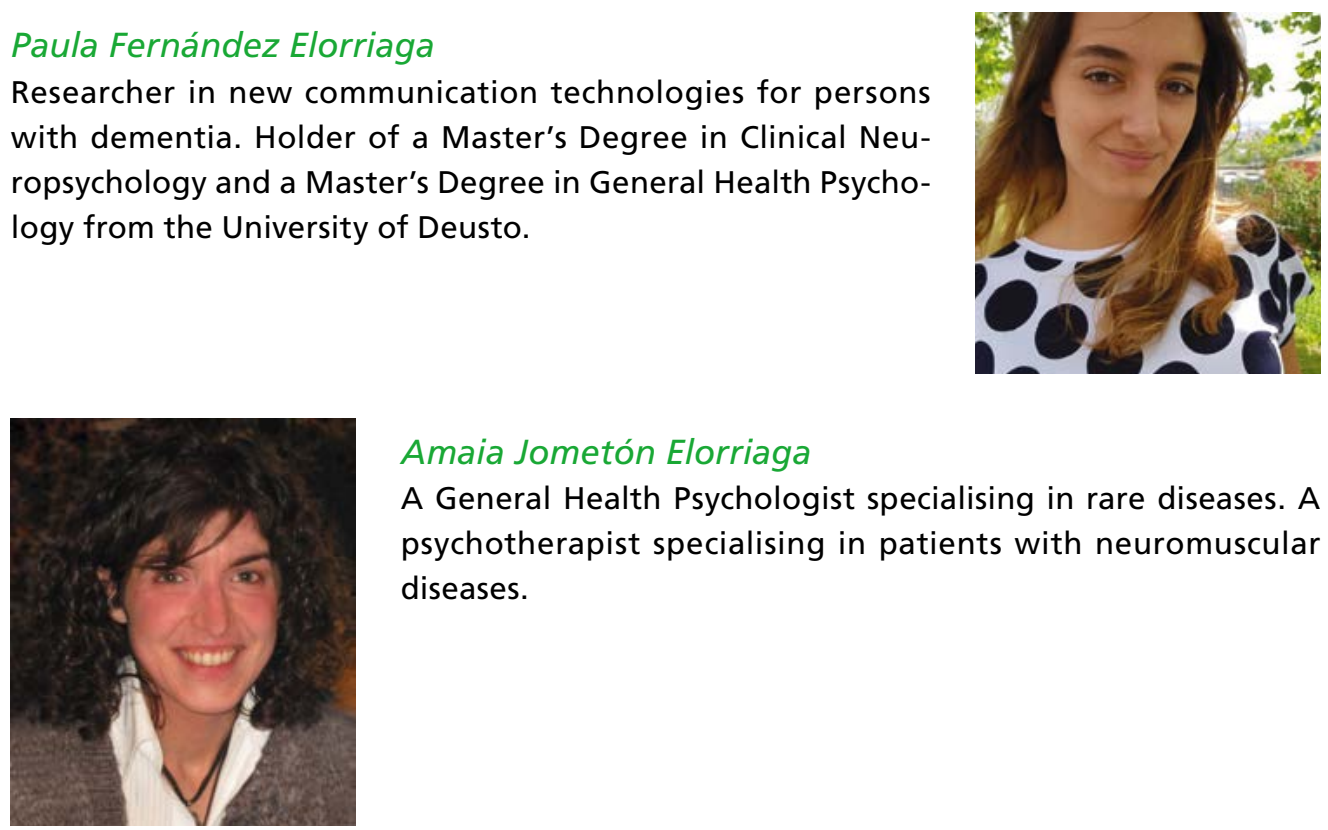

\section{Amaia Jometón Elorriaga}

A General Health Psychologist specialising in rare diseases. A psychotherapist specialising in patients with neuromuscular diseases.

\section{Imanol Amayra Caro}

Holder of the Chair of Psychology at the University of Deusto. Lead researcher on the Neuro-e-Motion team. Head of the Psychological Assessment, Personality and Treatment Department at the University of Deusto.

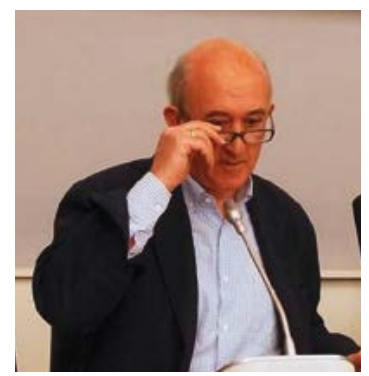






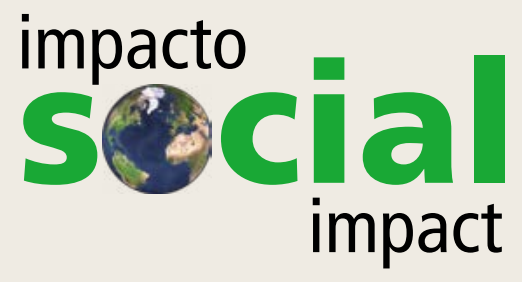

Nenra-e-Matian

enfermedades neuramusculares, neuromotoras y del neurodesarrollo

\section{ADELA EUSKAL HERRIA}

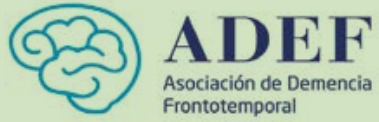

Frontotemporal

\section{g Deusto

